\title{
A Reactive Force Field for Aqueous-Calcium Carbonate Systems
}

Julian D. Gale, ${ }^{\dagger}$ Paolo Raiteri ${ }^{\dagger}$ and Adri C.T. van Duin ${ }^{\ddagger}$

${ }^{\dagger}$ Nanochemistry Research Institute, Department of Chemistry, Curtin University, PO Box U1987, Perth, WA 6845, Australia

${ }^{\ddagger}$ Department of Mechanical and Nuclear Engineering, The Pennsylvania State University, University Park, Pennsylvania 16802, USA

\begin{abstract}
A new reactive force field has been derived that allows the modelling of speciation in the aqueous-calcium carbonate system. Using the ReaxFF methodology, which has now been implemented in the program GULP, calcium has been simulated as a fixed charge di-cation species in both crystalline phases, such as calcite and aragonite, as well as in the solution phase. Excluding calcium from the charge equilibration process appears to have no adverse effects for the simulation of species relevant to the aqueous environment. Based on this model, the speciation of carbonic acid, bicarbonate and carbonate have been examined in microsolvated conditions, as well as bulk water. When immersed in a droplet of 98 water molecules and two hydronium ions, the carbonate ion is rapidly converted to bicarbonate, and ultimately carbonic acid, which is formed as the metastable cis-trans isomer under kinetic control. Both first principles and ReaxFF calculations exhibit the same behaviour, but the longer timescale accessible to the latter allows the diffusion of the carbonic acid to the surface of the water to be observed, where it is more stable at the interface. Calcium carbonate is also examined as ion pairs in solution for both $\mathrm{CaCO}_{3}{ }^{0}$ (aq) and $\mathrm{CaHCO}_{3}{ }_{(\mathrm{aq})}$, in addition to the (1014) surface in contact with water.
\end{abstract}




\section{Introduction}

Calcium carbonate is a commonly occurring mineral at the Earth's surface that is of particular significance. Being formed from dissolved calcium ions and carbon dioxide in seawater, it represents a natural material for carbon sequestration. While its formation is often beneficial, this process can also represent a technological problem as a result of scale formation in both vessels and pipes. The mechanisms by which calcium carbonate forms are particularly intriguing since it can exploit a nucleation pathway in which stable precursors are created prior to nucleation as amorphous calcium carbonate. ${ }^{1}$ This amorphous material subsequently undergoes a transformation to one of three possible crystalline polymorphs, calcite, aragonite or vaterite, in order of decreasing bulk stability at ambient conditions. Selective control of this interconversion lies at the heart of biomineralization - the process by which organisms fashion skeletal and other functional materials from minerals.

Aside from the complex nucleation behaviour of calcium carbonate, there is also interest in the mechanisms by which the crystalline phases grow. In the case of calcite in particular there is considerable information already, in that the rate of growth is typically nucleation limited under most conditions. The morphology of calcite is dominated by the stable basal (1014) surface, which has been extensively studied. Both experiment and theory agree that there is little association between ions and this flat surface as a result of the presence of two highly structured water layers creating a barrier to surface adsorption of other species. ${ }^{2,3}$ Consequently, growth is usually driven by the presence of defects, such as kink sites, or screw dislocations emanating from the surface. With the advent of atomic force microscopy, it has become possible to determine detailed information about surface processes, including step velocities and the influence of organic growth modifiers. ${ }^{4}$ Despite this wealth of information, there remain gaps in our knowledge regarding the atomic level detail of what is occurring at the carbonate-water interface.

One possible strategy to obtain further insights into the surfaces of carbonates is to employ atomistic simulation. Over the last few decades there have been numerous force field models developed designed to represent the bulk properties of calcium carbonate. $^{5,6}$ While most of these models accurately describe the structure and mechanical properties of calcite, and in some cases aragonite too, less attention has 
fallen on the ability to accurately reproduce the thermodynamic properties, especially in contact with aqueous interfaces. Recently we have performed an examination of the available force fields for the aqueous-calcium carbonate system and developed two new models that accurately capture the thermodynamics for this environment. ${ }^{7,8}$

A common feature of all current force fields, including the most recent ones, is that the carbonate anion is treated as a molecular entity (i.e. the bonding is predefined and must be preserved through out any simulation). For bulk carbonate phases, including amorphous calcium carbonate, this description is accurate and represents a reasonable choice. However, if the objective is to examine the growth carbonate materials then the situation is more complex due to the solution speciation of carbonate. When carbon dioxide dissolves in water the dominant species close to neutral $\mathrm{pH}$ is the bicarbonate anion. Although growth is normally conducted under basic conditions, the issue remains that bicarbonate may still prevail in solution, whereas carbonate is found in the crystal. The question therefore arises as to whether bicarbonate or carbonate is initially incorporated during growth, i.e. does proton transfer occur before or after addition to a kink site? Ideally one would attempt to answer such questions through the use of quantum mechanical simulations, so that the speciation is unconstrained. However, this remains a challenging undertaking, primarily because of the computational demands. There are also limitations to the accuracy of many density functionals in describing water leading to over-structuring at ambient conditions, though new functionals that incorporate long-range van der Waal's interactions may improve this situation. ${ }^{9}$

While the majority of force fields are unable to describe speciation effects, there are an increasing number of approaches for the inclusion of reactivity. One promising strategy is to allow changes in bonding through the use of multi-state empirical valence bond (MSEVB) theory. ${ }^{10}$ Here a coupling Hamiltonian is introduced that permits the mixing between two or more valence bond configurations for which conventional molecular mechanics descriptions can be utilised. This approach has already been applied to the study of an excess proton or hydroxyl group in liquid water, ${ }^{11}$ as well as proton transport in oxide materials. ${ }^{12}$ While potentially the most accurate approach, the downside for more complex systems is that the possible pathways for reaction must been predetermined so that a coupling expression can be derived and parameterised. 
An alternative approach to MSEVB theory is to design a force field that intrinsically does not make prior assumptions regarding the connectivity and that can mimic a quantum mechanical potential energy surface during reactive events. A natural basis to start from is a bond order formalism, such as those used to describe semiconductor or carbonaceous materials. Arguably the most sophisticated models to appear in this field, to date, are the $\mathrm{LCBOP}^{13}$ and $\mathrm{ReaxFF}^{14}$ force fields. Of these, only the latter approach has been widely parameterised for a range of materials and so we focus on this methodology. In ReaxFF, the treatment of van der Waal's and electrostatic interactions are included from the outset, as well as allowing for separate sigma, pi and pi-pi (single, double and triple) bond orders. In the present work, we explore the extension of the ReaxFF approach to the simulation of the aqueous calcium carbonate system and contrast the results with the findings of both first principles and conventional force field methods. Finally, the implications of the results for the bicarbonate-carbonate interconversion during growth will be considered.

\section{Methods}

The objective of the present work is to develop and apply the ReaxFF methodology to the simulation of the aqueous-calcium carbonate system. ReaxFF combines a bond order approach with a variable charge model. ${ }^{15}$ ReaxFF calculates Coulomb interactions between all atoms, including atoms sharing a bond. This enables the method to transition between covalent and ionic bond-types, which is important for the systems studied here. A full description of the functional form of the ReaxFF force field can be found elsewhere. ${ }^{16}$

For the purposes of the present work the ReaxFF methodology has been implemented within the program GULP. ${ }^{17,18}$ There are a few minor adjustments in functional form in that tapering functions have been applied to all interactions to ensure smooth truncation. At present only analytic first derivatives are included, though properties and phonons at the gamma point can be calculated through the use of central finite differences. Both conventional and domain decomposition algorithms have been implemented, with the latter being beneficial for the linear-scaling computation of large systems. 
The calculation of geometry-dependent charges by electronegativity equalisation can be performed either by direct matrix inversion or via iterative minimisation. Here the matrix of pair-wise Coulomb interactions is stored as a sparse matrix to conserve memory. The use of iterative methods is found to be superior in speed for all but the smallest systems, especially during molecular dynamics where the charge distribution from the previous time step represents an accurate initial guess for the subsequent minimisation. The use of five-point rational function extrapolation to propagate the charge distribution during dynamics was also examined. However, the reduction in number of iterations was found to be minimal, suggesting that initial convergence with preconditioning is fast and that the iteration count is dominated by the latter stages of minimisation. Convergence slow down due to the different length scales of charge oscillation is a common phenomenon in such problems. All aspects of the ReaxFF calculation, including the charge determination, are parallelised using MPI, within a replicated data paradigm at present.

The ReaxFF methodology has already been parameterised for a wide range of systems including hydrocarbons, ${ }^{16}$ metal oxides ${ }^{19}$ and hydrides. ${ }^{20}$ Recently, the parameterization has been extended to include water and aqueous species. In the present work we adopt these parameters for water largely unchanged, as well as those for carbon. Hence, the challenge for this study is to include calcium into the parameterization. Traditionally, the parameters for any new element have been determined by fitting to an extensive database of results obtained from ab initio quantum mechanical calculations. While such a complete fitting procedure is supported by the implementation in GULP, we have decided to take an alternative approach. For the purposes of the present study, calcium is always present as a dication, i.e. $\mathrm{Ca}^{+2}$, regardless of whether it is in aqueous solution or within the bulk of a crystalline phase of calcium carbonate. Given this intransigent ionic behaviour during crystal growth, it is unnecessary to utilise a fully reactive description for calcium that is capable of spanning calcium metal through to the cationic state. Indeed, any attempt to derive electronegativity parameters that will yield the necessary electrostatic contribution to calcium's interactions will be fraught with difficulty. Anything less than a charge of close to +2 would result in under binding of water to calcium when solvated in solution, unless corrected by a covalent bond order contribution. However, to parameterize a charge equilibration model to yield close to the formal ionic state, but not to exceed it, requires the incorporation of shell structure. To do this implies a 
non-quadratic dependence of energy on charge, which in turn leads to the need to iteratively solve for the charges in all cases (i.e. not only do the atomic charges have to be iteratively minimised, but the matrix of Coulomb interactions needs to be partially recomputed at every iteration).

To resolve the above situation for calcium, we adopt a formal ionic description for this species as a fixed charged $\mathrm{Ca}^{2+}$ species. For calcium carbonate the corresponding two electrons required to maintain neutrality are added to the charge equilibration for the carbonate anion. Rather than treating calcium as a point ion, we adopt the same, screened Coulomb interaction as employed generally within ReaxFF;

$$
U_{\text {Coulomb }}^{i j}=\frac{q_{i} q_{j}}{\left[r_{i j}^{3}+\left(1 / \gamma_{i j}\right)^{3}\right]^{1 / 3}} f\left(r_{i j}\right)
$$

where $f\left(r_{i j}\right)$ is a taper function to ensure that the interaction goes smoothly to zero at the cut-off distance of $10 \AA$, and atomic units are employed. Given that the screening parameter is determined via the combination rule;

$$
\gamma_{i j}=\sqrt{\gamma_{i} \gamma_{j}}
$$

this introduces a single fitting parameter for calcium, prior to consideration of pairwise interactions.

In addition to the electrostatic contribution, it is necessary to represent the shortrange repulsion between calcium and other species. In the spirit of a reactive force field, these interactions must be universal regardless of the chemical environment. Hence, the only contributions to be determined are the pair-wise repulsions for $\mathrm{Ca}-\mathrm{O}$, $\mathrm{Ca}-\mathrm{H}$ and $\mathrm{Ca}-\mathrm{C}$. We assume that the van der Waal's attraction for the calcium dication will be negligibly small due to its low polarisability. Furthermore, it is assumed that many-body contributions will also be insignificant in this case. Support for this comes from our recent conventional force field in which two-body interactions alone were sufficient to describe calcium ions. ${ }^{7}$

Based on consideration of configurations relevant to the aqueous-calcium carbonate system alone, it is unlikely that the $\mathrm{Ca}-\mathrm{H}$ and $\mathrm{Ca}-\mathrm{C}$ short-range repulsive 
interactions would be well defined. In both cases, electrostatic repulsion would dominate due to the like-signed charges observed. To overcome this, the parameters for these interactions have been fitted to the experimental structures of calcium carbide $^{21}\left(\mathrm{CaC}_{2}\right)$ and calcium hydride ${ }^{22}\left(\mathrm{CaH}_{2}\right)$ in which carbon and hydrogen are in anionic states. All fits are performed using the relaxed fitting algorithm, in which the least squares objective function is computed based on the changes in structure during optimisation, rather than the forces/stresses at the experimental configuration.

Having determined the $\mathrm{Ca}-\mathrm{H}$ and $\mathrm{Ca}-\mathrm{C}$ interactions as above, these terms were kept fixed. The remaining $\mathrm{Ca}-\mathrm{O}$ interaction was determined by fitting to a combination of data, including the experimental structures for calcite, ${ }^{23}$ portlandite $\left(\mathrm{Ca}(\mathrm{OH})_{2}\right),{ }^{24}$ calcium oxide $(\mathrm{CaO})$ and $a b$ initio structure and binding energy information for hexa-aquo calcium $\left(\mathrm{Ca}\left(\mathrm{H}_{2} \mathrm{O}\right)_{6}{ }^{2+}\right)$. The Coulomb screening parameter for $\mathrm{Ca}$ was also fitted to this data set. Because the $\mathrm{Ca}-\mathrm{H}$ and $\mathrm{Ca}-\mathrm{C}$ interactions are also influenced by the screening term, the two stages of fitting were repeated iteratively until a consistent value of $\gamma_{\mathrm{Ca}}$ was obtained.

During simulations of speciation in bulk water with the reactive force field it was found to be necessary to make a small change to the ReaxFF parameters for water. It was observed that there is a tendency when some species, such as the hydroxyl anion, are present to form hydrogen peroxides or even peroxide groups bound to carbon. Given that there is no evidence that molecules of this nature are relevant to the aqueous chemistry of carbonate in the absence of a redox bias, the formation of peroxides has been suppressed by zeroing the bond strength for $\mathrm{O}-\mathrm{O}$ interactions. This has negligible influence on the properties of water or other molecules of interest for the $\mathrm{O}-\mathrm{O}$ distances sampled. The probable reason for the spurious formation of peroxides in the presence of $\mathrm{OH}^{-}$is that the standard electronegativity equalisation model fails to fully localise the negative charge on the anion, ${ }^{25}$ therefore leading to it having a character that is intermediate between the desired state and a hydroxyl radical.

In order to benchmark the results of the ReaxFF force field for speciation in water, a number of quantum mechanical calculations were performed for comparison. For small gas phase species calculations were performed at the M06-L/aug-cc-pvtz level of theory ${ }^{26}$ using the NWChem program. ${ }^{27}$ For larger systems, the SIESTA methodology and program was employed. ${ }^{28}$ Here calculations were carried out using density functional theory within the Generalized Gradient Approximation, based on 
the PBE functional. ${ }^{29}$ Non-local norm conserving pseudopotentials of the modified Troullier-Martins form ${ }^{30}$ were used to represent the nuclei and core electrons while valence Kohn-Sham states were expanded in a basis set of strictly localised pseudo atomic orbitals (PAOs). The confinement radii for oxygen, and carbon were set at 7 and 6 Bohr, respectively, while hydrogen had a radius of 7 Bohr for the 1s PAO and 3.5 Bohr for the polarisation functions. Soft confinement was used to ensure the smooth decay of the basis functions to the cut-off with a potential of $100 \mathrm{Ry}$ and inner radius at 0.95 of the orbital cut-off. The basis set was of DZDP quality (double-zeta, doubly polarised), with the addition of d-functions on hydrogen, using a split-norm of 0.25 for carbon and oxygen, but 0.5 for hydrogen. The density was expanded in an auxiliary basis set of a uniform Cartesian mesh with a kinetic energy cut-off of 250 Ry.

Molecular dynamics simulations were performed with the ReaxFF force field using the method described by Raiteri et al. ${ }^{12}$ in which a stochastic thermostat and barostat are utilised in the $N P T$ ensemble. For liquid phase simulations the cell was constrained to vary isotropically, while for surfaces and interfaces orthorhombic constraints were imposed to prevent shearing. Unless otherwise stated, the temperature was specified to be $298.15 \mathrm{~K}$, with no external pressure, and the thermostat/barostat relaxation times both set to $0.01 \mathrm{ps}$. A time step of $0.5 \mathrm{fs}$ was used with adiabatic treatment of the charge distribution. Systems were equilibrated for 300 ps, during which velocity rescaling was applied, followed by 1 ns of production during which the trajectory and ensemble averages were collected.

We note that some molecular dynamics simulations of carbonate species in water can lead to the formation of carbon dioxide, as is the prerogative of a reactive force field. Physically there is nothing wrong with this since carbon dioxide can exist in water, as evidenced by the formation of clathrates. However, it serves to highlight the need to be careful regarding the initialisation of the simulations to target the state of interest. For this reason, molecular dynamics runs were typically equilibrated first with harmonic restraints on key bonds to allow any excess energy to be dissipated through velocity rescaling rather than chemical reactivity. After this, simulations were further equilibrated without any restraints prior to production. 


\section{Results and discussion}

1) Bulk phases

The final fitted parameters, determined using the procedure described above, are given in Table 1, while the complete force field library for GULP is available as supporting material. For the solid phases, the calculated values are compared against the experimental data used to derive the parameterisation in Table 2. In the case of the main target polymorph for $\mathrm{CaCO}_{3}$, calcite, reproduction of the structure is very good with the errors being of a similar magnitude to those obtained from conventional ionic model descriptions, despite the more limited number of fitting parameters. The $c$ axis of the hexagonal cell is particularly well reproduced, with an overestimation of only $0.5 \%$, while the $\mathrm{C}-\mathrm{O}$ bond length of the carbonate anion is almost exactly captured as the only symmetry unconstrained geometric parameter for this species. Similarly, the bulk modulus, based on the Reuss definition, agrees well with experiment ${ }^{31}$ and also values from density functional calculations. However, the anisotropy of the elastic behaviour deviates from that found experimentally with the elastic constants in the $a b$ plane being too high, while $\mathrm{C}_{33}$ is $74 \mathrm{GPa}$, which is $13 \%$ below the experimental value. $^{32}$

For the second crystalline polymorph of calcium carbonate, aragonite, the errors in the structure are larger. The origin of this lies in the phonon spectrum of the optimised experimental structure, in that there are 3 imaginary modes at the centre of the Brillouin zone. These unstable modes correspond to the rotation of the carbonate group within the aragonite cell, thus leading to an anisotropic distortion of the cell relative to experiment. It should be noted that this failing is not uncommon for conventional force fields for $\mathrm{CaCO}_{3}$ with the widely used model of Freeman et al. ${ }^{33}$ also suffering from this problem.

One of the key points of interest regarding pure calcium carbonate phases is the thermodynamics of the polymorphs. Experimentally, aragonite is the lower enthalpy phase by $0.44 \mathrm{~kJ} / \mathrm{mol}$ and is the stable polymorph at moderately elevated pressures. However, under standard conditions calcite has the lower free energy by 0.84 $\mathrm{kJ} / \mathrm{mol}^{34}$ Most literature force fields struggle to describe this energy difference and yield values that are an order of magnitude too large and/or have the wrong sign. Recently, we have developed both rigid $^{7}$ and flexible ${ }^{8}$ carbonate models that 
accurately reproduce the free energy difference between calcite and aragonite at $25^{\circ} \mathrm{C}$, but these force fields do not allow for carbonate reactivity. The parameterisation developed in this work gives a free energy difference between calcite and aragonite of $0.84 \mathrm{~kJ} / \mathrm{mol}$ in favour of calcite at $298 \mathrm{~K}$, based on the lattice dynamical contribution from the phonons. This perfect agreement with experiment is coincidental as the energy difference was not fitted, and the structure of aragonite differs from that found experimentally. Furthermore, because the sampling of phonons across the Brillouin zone is not presently implemented there will also be a small numerical error in this value. It should also be noted that the internal energy and enthalpy differences are 1.64 and $1.55 \mathrm{~kJ} / \mathrm{mol}$, respectively, also in favour of calcite and thus the experimental variation with temperature would not be reproduced. It is interesting to note that all force fields that predict the wrong carbonate orientation in aragonite also yield a more favourable enthalpy for calcite than free energy, in contrast to experiment. In the current context of being able to simulate aqueous carbonate systems, the prediction of the correct thermodynamics close to standard conditions is the most important characteristic, even if there may be limitations in more extreme regimes. In addition, the thermodynamic properties of the reactive model for calcium carbonate are as good, if not better than, most literature force fields, excluding those recently developed to target accurate free energies.

Moving away from the carbonate phases, the reproduction of cell parameters exhibits variable degrees of success, though is respectable for only six fitted quantities when considering a wide range of chemical environments, such as hydrogen going from proton-like states to anionic hydride. Arguably the most significant structural error is the underestimation of the cubic cell parameter for the rock salt structured $\mathrm{CaO}$. Here the use of a formal charge on calcium constrains the oxygen to be an $\mathrm{O}^{2-}$ anion. This extreme situation is outside the parameterisation range for oxygen, which was trained against more covalent environments. Hence, this deviation is perhaps to be expected. Although ReaxFF has been previously applied to oxides, such as $\mathrm{ZnO}$ and $\mathrm{BaZrO}_{3},{ }^{19,35}$ this was for a different set of parameters to those that are derived for water. In none of these aforementioned oxides from prior studies was oxygen six coordinate or formally charged.

Portlandite, $\mathrm{Ca}(\mathrm{OH})_{2}$, is one of the most difficult systems being a layered structure and so a small error can lead to considerable change parallel to the $c$ axis. Despite this, the cell is only overestimated by $6.9 \%$ in the interlayer direction, while 
the layers are too small in the $a b$ plane by $4.6 \%$. This is partially reflected in the elastic constants where $C_{11}$ is computed to be $104.4 \mathrm{GPa}$, as opposed to $102 \pm 2 \mathrm{GPa}$ as measured. ${ }^{36}$ In the $c$ direction, the layers are found to be over bound in spite of the elongated lattice parameter, with $C_{33}$ being $105 \mathrm{GPa}$ instead of $33.6 \pm 0.7 \mathrm{GPa}$. Note that for this material all calculations are performed under an applied pressure of 0.07 GPa to match the experimental conditions. Recent periodic ab initio calculations ${ }^{37}$ on portlandite at the B3LYP and B3LYP-D levels found similar errors to those above for the interlayer spacing in the absence of the dispersion correction and computed an interlayer interaction energy of $-22.7 \mathrm{~kJ} / \mathrm{mol}$. This confirms the overbinding for the reactive force field, which gives $-38.6 \mathrm{~kJ} / \mathrm{mol}$ for the same quantity.

Before leaving the training set of solids, the remaining property we can consider is the charge distribution in the materials, as given in Table 3. As would be expected, the carbonate groups in both calcite and aragonite have similar charges on carbon and oxygen, with the latter polymorph being just marginally more ionic. The charge on oxygen in both cases is just over -0.93 a.u., which is just slightly below (in absolute magnitude) the values found in the majority of fixed charged carbonate force fields, where the oxygen is typically in the range -0.99 to -1.12 a.u.. For portlandite, the hydrogen charge is slightly below values obtained from Mulliken population analysis in $a b$ initio calculations, which are between +0.347 for Hartree-Fock and +0.331 for B3LYP. ${ }^{38}$ However, Mulliken charges are not always a good guide to the best atomcentred monopole that captures interaction energies.

2) Hydration of calcium ion by water

The hydration of calcium in water has been extensively studied, both experimentally and theoretically. In solution, calcium exhibits a variable coordination number that fluctuates about an average just above 7 water molecules, ${ }^{39}$ with relatively rapid exchange occurring. ${ }^{40}$ Aside from the solution studies, there have also been numerous quantum mechanical examinations to determine the successive binding energies of water molecules in the gas phase. This latter data provides an ideal basis for evaluation of the present force field model given the well-characterised nature of the states involved. 
In Table 4 we report the adsorption energies of water molecules to $\mathrm{Ca}\left(\mathrm{H}_{2} \mathrm{O}\right)_{\mathrm{n}-1}{ }^{2+}$ in vacuo for $\mathrm{n}=1$ to 7 and compare these values against other theoretical estimates. All of the models are consistent in exhibiting a substantial decrease in the exothermicity of water binding when going beyond octahedral coordination. Examining the broader variation of energies, here the benefit of a model that employs environment-dependent charges becomes apparent. In a fixed charged force field, even when including a shell model for polarisation, the variation in binding energy between the addition of the first and last water is underestimated; in other words, such models usually have to be fitted to target the binding energy of water close to the maximum coordination number observed. By using variable charges, the model is better able to capture the many-body contribution to water binding and can therefore attempt to describe the extremes of coordination number.

Aside from the individual water binding energies, the cumulative sum of this quantity up to the probable coordination number of calcium in solution is also important, since this will relate to the enthalpy of solvation in aqueous solution. In Table 4, the sum of the hydration energies up to both 6 and 7 waters are given. While the individual contributions may vary, both the flexible unreactive and reactive force fields agree quite well on the total hydration energy of calcium. Because the flexible unreactive model is fitted to reproduce the experimental free energy of solvation of calcium in bulk water, this indicates that the present reactive model should also perform well in this regard. The cumulative hydration energy from Bakó et al. ${ }^{41}$ is less exothermic than those from the flexible unreactive and reactive force fields, which is consistent with the under binding tendency often associated with the use of a GGA functional within Kohn-Sham density functional theory.

Turning to consider the geometrical properties of the water clusters, Bakó et al. find a lengthening of the optimized $\mathrm{Ca}-\mathrm{O}$ distance as the number of waters increases. Here we find the same trend as this distance increases from 2.245 (2.248) to 2.406 (2.422) $\AA$ in going from one to six water molecules (here the values in parenthesis are those of Bakó et al.).

The key point established in this section is that despite the fixed charge description of calcium as a +2 cation, the solvation properties in water appear to be accurately captured. The polarisation of the water alone is sufficient to mimic much of the cooperative interaction between neighbouring molecules without charge transfer to calcium. Indeed, it is possible that including calcium within the charge 
equilibration would lead to a worse description of hydration since the invariably lower charge from such a procedure would cause an underestimation of the electrostatic contribution to the water binding strength. Despite this, a previous study with ReaxFF has successfully used full charge equilibration to study the $\mathrm{Cu}^{2+}$ cation in water. ${ }^{42}$ Here the water binding energies for $\mathrm{Cu}\left(\mathrm{H}_{2} \mathrm{O}\right)_{\mathrm{n}}{ }^{2+}$ of a QM training set are reproduced to within an average unsigned deviation of $8 \mathrm{~kJ} / \mathrm{mol}$, if the $\mathrm{n}=1$ and 2 cases are excluded, which is comparable to the present work. It should be noted that the individual water binding energies were not fitted for the case of calcium, which again points to the quality of the hydration behaviour being more intrinsic to the water model than the metal-water interaction.

\section{3) Gas phase reactivity}

The ReaxFF force field for gas phase chemistry of organics has already been documented in previous works on this topic ${ }^{16}$ and the present work utilises existing parameters with only minor changes. Hence we shall only briefly consider a few aspects of the gas phase chemistry of species relevant to carbonate equilibria. Of these, the most important are carbonic acid $\left(\mathrm{H}_{2} \mathrm{CO}_{3}\right)$, the bicarbonate anion $\left(\mathrm{HCO}_{3}{ }^{-}\right)$ and the carbonate anion $\left(\mathrm{CO}_{3}{ }^{2-}\right)$, as they are connected by proton transfer reactions in solution, plus carbon dioxide and water/hydroxide as reactants that can also form these species. When in vacuo the carbonate ion itself is not significant due to the intrinsic instability of the di-anion and thus consideration of this state is deferred until the aqueous phase is considered.

Starting with the neutral species, ReaxFF generally gives a good reproduction of most structural properties. For example, the C-O bond length in carbon dioxide is $1.185 \AA$, as compared to $1.180 \AA$ at the MP2/aug-cc-pvdz level of theory. ${ }^{43}$ These values are longer than the experimental value of $1.162 \AA$, with the overestimation by ReaxFF having its origin in the fact that the parameters are determined through the use of quantum mechanical energy surfaces rather than experimental ones. The C-O bond lengths in carbonic acid are slightly longer than the corresponding ab initio values at 1.231 (1.218) and 1.377 (1.351) $\AA$, while the O-H distances of $0.956(0.971)$ $\AA$ are slightly too short (MP2 values in parenthesis ${ }^{43}$ ).

Carbonic acid has three geometric isomers depend on the cis or trans orientation of each hydroxyl group with respect to the other. Previous theoretical works ${ }^{44}$ have 
established that the trans-trans ( $\mathrm{tt}$ ) configuration is the most stable, closely followed by the cis-trans (ct) one, while the cis-cis (cc) isomer is notably higher in energy. This trend is reproduced by the reactive force field with $\mathrm{tt}-\mathrm{H}_{2} \mathrm{CO}_{3}$ being $2.0 \mathrm{~kJ} / \mathrm{mol}$ lower in energy than ct $-\mathrm{H}_{2} \mathrm{CO}_{3}$ and $28.1 \mathrm{~kJ} / \mathrm{mol}$ more stable than the remaining isomer.

Turning to consider reaction energetics, the first process of interest is reaction of carbon dioxide with water to form carbonic acid. Liedl et al. have computed the energy of formation of carbonic acid from water and $\mathrm{CO}_{2}$ to lie between +16.7 and $+20.9 \mathrm{~kJ} / \mathrm{mol}$ in the gas phase. ${ }^{45}$ On top of this is a zero point energy correction of $+17.6 \mathrm{~kJ} / \mathrm{mol}$, which is less sensitive to the nature of the calculation, thus placing an upper bound on the internal energy change of $+38.5 \mathrm{~kJ} / \mathrm{mol}$. In contrast, the ReaxFF results yield a much higher value of $+127 \mathrm{~kJ} / \mathrm{mol}$. This suggests that caution must be exercised when considering reactions that change the coordination number of the carbon centre to oxygen.

A second reaction, and one that is more critical to the study of carbonates in contact with water, is the dissociation of carbonic acid to bicarbonate and a proton. The computed energetics of this process as compared to quantum mechanical values are given in Table 5 for three reactions depending on the final state of the proton. Here we compute all energies with respect to the trans-trans isomer of carbonic acid and consider only the total energy differences without zero point energy correction for consistency. As can be seen, the energy of proton removal from carbonic acid in the gas phase is substantially underestimated when forming $\mathrm{H}_{(\mathrm{g})}^{+}$. However, when water is included in the reaction to create a hydronium ion the situation is considerably improved. When two waters are present then there is good agreement. It should be noted that the stable state for $\mathrm{H}_{5} \mathrm{O}_{2}{ }^{+}$within the reactive force field is an asymmetric Zundel structure, contrary to accurate $a b$ initio results. ${ }^{46}$ However, the symmetric form is a transition state that is only $5.2 \mathrm{~kJ} / \mathrm{mol}$ higher in energy, consistent with the finding that the potential energy surface for the shared hydrogen is relatively flat.

The reason for the improved energetics of proton transfer as the degree of solvation of the proton increases reflects the fact that the water parameters were derived, at least in part, for this limit. One possible source of the discrepancy for the isolated proton is the electronegativity equalisation method. Although the ionization energy of hydrogen is only underestimated by $27 \mathrm{~kJ} / \mathrm{mol}$ in ReaxFF, the harmonic interpolation between charge states is not truly realistic and so can lead to errors in the energy when comparing states of different charge. When the proton is bound to water 
molecules this effect is minimised as the charge adopted in the Zundel ion ( +0.42 a.u.) is similar to that in carbonic acid ( +0.39 a.u.).

Although not relevant to aqueous carbonate chemistry, we can also examine carbonic acid acting as a base to form the species $\mathrm{C}(\mathrm{OH})_{3}{ }^{+}$. Using the anti isomer of Andrei et al., ${ }^{47}$ the computed proton affinity is $123.4 \mathrm{~kJ} / \mathrm{mol}$ with ReaxFF, versus 84.6 $\mathrm{kJ} / \mathrm{mol}$ at the M06-L level. While there is some discrepancy, this confirms that carbonic acid is only a weak base and therefore will not offer an invalid reactive channel during simulations in water. Furthermore, the ReaxFF parameterisation correctly identifies that protonated carbonic acid is less stable that carbon dioxide hydrogen bonded to a hydronium ion, though the energy difference is exaggerated (74 vs $18 \mathrm{~kJ} / \mathrm{mol}$ in the work of Andrei et al.)

4) Static and dynamical exploration of the microsolvation of carbonate.

Given the current level of interest in carbon dioxide sequestration and the impact of this gas on oceans, there has been extensive research into the equilibria when this molecule comes in contact with water. ${ }^{48,49}$ Carbon dioxide can remain in the molecular state in water, especially when trapped within clathrates, but under many conditions will also react with the surrounding water. Initially, $\mathrm{CO}_{2(\mathrm{aq})}$ is proposed to form carbonic acid $\left(\mathrm{H}_{2} \mathrm{CO}_{3}\right)$ as a short-lived intermediate species, which rapidly dissociates to form the bicarbonate anion in the region of neutral $\mathrm{pH}$, though a recent theoretical work has proposed that the formation of bicarbonate occurs directly without going via carbonic acid. ${ }^{50}$

Before considering the behaviour of carbonate in bulk water, it is interesting to examine the static properties and dynamical evolution of species in the presence of smaller quantities of water. The nature of such species in the absence of excess water is of interest given that carbonic acid has been suggested to be present in extraterrestrial environments, ${ }^{51}$ but is unstable when water is present.

To probe carbonate reactivity when microsolvated we have performed equivalent molecular dynamics simulations with both first principles techniques and the present reactive force field. Here the quantum mechanical results are obtained using the SIESTA methodology with the PBE functional. Both the quantum and classical simulations commence from the same set of atomic positions and are performed at the 
same temperature, though we note the initial velocities are randomly chosen and therefore differ.

In the first simulation, a symmetrically arranged configuration of eight water molecules hydrogen bonded to a carbonate anion and each other is chosen. To maintain charge neutrality, two protons are added to create solvated hydroxonium cations within the cluster. The starting configuration is illustrated in Figure 1a. Once the dynamics commences at $300 \mathrm{~K}$, proton transfer rapidly occurs from one hydronium, via an intermediate water, to the carbonate to generate the bicarbonate species. Before long the second proton also transfers to bicarbonate (Fig. 1c), thereby forming carbonic acid. Congruent behaviour is observed in both the quantum and classical runs, except that the timescale for both transfer events differs; proton migration occurs more rapidly in the quantum mechanical simulations. While the first principles results could be considered to be the more accurate, it is known that GGA exchange-correlation functionals can lead to underestimation of proton transfer barriers. ${ }^{52}$ Consequently, the reactive force field deviates towards what is, in all probability, the experimental side of the GGA result.

To examine further the above proton transfer behaviour, the same procedure was followed to probe the lifetime of the carbonate anion in a small droplet consisting of 100 water molecules. At the outset of the molecular dynamics the carbonate ion was positioned in the centre of the droplet while the counter balancing hydronium ions were placed at diametrically opposite sides of the surface in an attempt to maximise the lifetime of the anion and minimise any initial dipole moment. The first principles simulation was performed for $2.5 \mathrm{ps}$, while the less expensive reactive force field was allowed to continue to $0.5 \mathrm{~ns}$. Although comparisons of timings should be regarded with caution since they are sensitive to the nature of the machine, compilers, etc, the ReaxFF approach is of the order of 50,000 times faster for this system.

In the first principles simulation, one of the protons rapidly transfers (within the first $0.1 \mathrm{ps}$ ), via two Grotthuss diffusion events, from the surface of the droplet to the carbonate ion at the centre to create bicarbonate. The configuration at the point of the second Grotthuss jump is shown in Figure 2. The second proton undergoes multiple jumps between molecules at the surface of the water before migrating to the subsurface layer of water where it forms a hydronium ion in contact with the bicarbonate anion. There are multiple proton fluctuations along the $\mathrm{H}_{3} \mathrm{O}^{+} \ldots \mathrm{HCO}_{3}{ }^{-}$hydrogen bond before finally the vibrational energy is sufficiently dissipated in the carbonic acid 
state to trap the proton in this minimum. After this event, which occurs after $2 \mathrm{ps,}$ both protons of the carbonic acid exhibit strong fluctuations towards the waters to which they donate hydrogen bonds. Although no proton transfer actually occurs, it is clear that carbonic acid is only marginally more stable than the hydroniumbicarbonate complex. All of this behaviour is consistent with the findings of Kumar et $a l .{ }^{53}$ who performed Car-Parrinello molecular dynamics on carbonate species in bulk water and observed a tendency for carbonic acid to dissociate for short periods.

As further evidence for the similar stability of the hydronium-bicarbonate cluster, two additional short simulations of 10 ps were run for the smaller eight water clusters at temperatures of 100 and $200 \mathrm{~K}$. At the lower temperature, proton transfer occurs to form bicarbonate after $0.075 \mathrm{ps}$, but there after the hydronium-bicarbonate species remains stable for the duration of the simulation indicating that there is a nonnegligible barrier even at this low level of hydration. At $200 \mathrm{~K}$, the bicarbonate takes longer to form $(0.13 \mathrm{ps})$ as the thermal motion begins to disrupt the ordered hydrogenbonding pattern of the starting configuration, thereby slowing proton transfer. Again, there is no formation of carbonic acid observed during 10 ps suggesting that the final proton transfer is more strongly activated in comparison to the formation of bicarbonate (Fig. 1b).

In the equivalent force field simulation of the larger water cluster, again a similar sequence of events occurs leading to the ultimate formation of carbonic acid via bicarbonate. As would be anticipated from the case of carbonate with eight water molecules, the proton transfer events are discernibly slower with the reactivity force field with both protons exhibiting several diffusion events through the water of solvation before either one reacts with carbonate. The $\mathrm{H}_{3} \mathrm{O}^{+} \ldots . \mathrm{HCO}_{3}{ }^{-}$complex also appears to be less stable; once carbonic acid is formed there are fewer attempted proton transfer events.

Because of the significantly greater length of the reactive force field simulation it is possible to observe another event that could not been seen on the short timescale accessible to first principles techniques. Over the course of the $0.5 \mathrm{~ns}$ of dynamics, the carbonic acid diffuses from the centre of the droplet to the surface where it is expelled from the water and becomes an adsorbed species. This indicates that the hydrogen bonding between carbonic acid and water is weaker than that for water-water and thus the relative hydrophobicity causes it to be more stable at the droplet surface. A similar 
effect has been observed for hydroxyl groups due to the absence of hydrogen bond donation by $\mathrm{OH}^{-}{ }^{54}$

A further interesting observation is that in both the first principles and ReaxFF simulations the carbonic acid forms, and remains in, the cis-trans isomer, which is slight metastable with respect to the trans-trans hydrogen conformation. This phenomenon can be explained by considering the dipole moment of the bicarbonate anion; this favours the incoming proton approaching from the side of the opposite oxygen to the existing hydrogen. On the timescale accessible within the current simulations there is no interconversion of the isomers of carbonic acid, both due to the intrinsic torsional barrier and also because the surrounding hydrogen bond network further increases this, and thus $\mathrm{H}_{2} \mathrm{CO}_{3}$ remains locked into the cis-trans isomer.

5) Ion pairing in bulk water.

Solution speciation of calcium and carbonate ions is crucial for understanding the growth mechanisms of this important family of resulting minerals. A key factor is the degree to which the ions associate in solution and the protonation state of the carbonate when this occurs. Both the $\mathrm{CaCO}_{3}{ }^{0}$ (aq) and $\mathrm{CaHCO}_{3}{ }^{+}$(aq) ion pairs are proposed to exist and their equilibrium constants for formation measured experimentally, with $\log K$ values of 3.2 and 1.14, respectively, under standard conditions. This implies standard free energies of formation of -18.3 and $-6.5 \mathrm{~kJ} / \mathrm{mol}$, again respectively. Hence, depending on the ion concentrations, ion pairing should be expected to occur.

To probe the state of the ions in solution we have performed a series of molecular dynamics simulations of the ions in a periodic cell containing 256 molecules of water at $298.15 \mathrm{~K}$. First of all, the calcium and carbonate ions were placed at some distance from each other within the cell. Here the ions gradually diffused towards each other, presumably as a result of the electrostatic interaction given that the screening will not approach the macroscopic dielectric constant at the length scale of the simulation. While the ions reach the state in which they are solvent separated from each other the tight ion pair is not formed on the timescale examined. This suggests that the barrier to desolvation of calcium is higher in the present force field than for our unreactive model where the activation energies are of the same order as ambient thermal energy and thus ion pairing is fast. 
In order to explore the contact ion pair configuration, the calcium and carbonate ions were placed together within the simulation. As expected, the ions remained coordinated for the duration of the simulation. To try to quantify the stability of the ion pair we can compare the relative energies of the separated and contact ion pair based on the averaged values from the simulations. For the contact ion pair the system is in a steady state for much of the period sampled and so the averaged quantities remain relatively constant. In contrast, the solvent separated case explores a number of different minima as a function of the number of water molecules between the cation and anion. As a result, the potential energy shows a degree of drift in the average according to the current state. Due to this, and because of the intrinsic statistical nature of the runs, there will be a considerable uncertainty in this energy difference. Accordingly, the enthalpy difference varies between approximately 35 and $60 \mathrm{~kJ} / \mathrm{mol}$ in favour of the contact ion pair with the lower bound corresponding to the point where the solvent separated ion pair only has a single shell of water between the ions. Experimentally, the enthalpy of dissociation of the ion pair under standard conditions is reported to be $42 \mathrm{~kJ} / \mathrm{mol}$ and therefore is consistent with current findings even if it is not possible to make an absolutely quantitative comparison. To further complicate matters, it has now been demonstrated that what was widely reported as the experimental thermodynamics of ion pairing almost certainly corresponds to the formation of pre-nucleation clusters instead, ${ }^{1}$ thus making the direct comparison even more difficult.

Based on earlier simulations, two binding configurations are believed to exist for the ion pair in which there is either monodentate or bidentate coordination. ${ }^{55}$ Different models favour the two binding modes. Force fields where the solubility of calcite is underestimated have been shown to favour bidentate coordination, due to the overestimation of the strength of the $\mathrm{Ca}-\mathrm{CO}_{3}$ interaction relative to that of the ions with water. Conversely, those that have their solubility close to the experimental value prefer a monodentate ion pair as this allows greater solvation by water. Our simulations exhibit almost exclusively monodentate coordination indicating that the balance of interactions is reasonable. Over the lifetime of the simulation all three oxygens are coordinated to calcium at some stage, with the oxygen exchange occurring via the bidentate geometry as either a transition state or a short-lived metastable configuration. A total of 3 clear exchange events are observed during $1 \mathrm{~ns}$ 
at intervals of a few hundred picoseconds. This is consistent with the order of magnitude of rate of water exchange for calcium ions measured experimentally.

Despite the lack of evidence for protonation of the carbonate in the ion pair, it is possible that this could be due to the magnitude of the activation barrier leading to the event not being seen on the nanosecond timescale. To confirm the preferred protonation state, a simulation was run commencing from the $\mathrm{CaHCO}_{3}{ }^{+}$ion pair. Charge neutrality was achieved by deprotonating a water molecule on the opposite side of the calcium ion to the bicarbonate anion to create a $\mathrm{CaOH}^{+}$species, which is also believed to be important in the solution equilibria under basic conditions. To maximise the probability of attaining a local minimum corresponding to this state, harmonic restraints were applied to the $\mathrm{O}-\mathrm{H}$ bonds of the hydroxyl and bicarbonate during the equilibration phase of the simulation to allow the solvating water molecules to accommodate this geometry. Within 4 ps of removal of the restraints the hydroxyl group coordinated to calcium was protonated by a neighbouring water and the hydroxyl group effectively diffuses away from the ion pair by a Grotthuss mechanism. After 950 ps the hydroxide anion has migrated to be adjacent to the hydrogen of the bicarbonate anion, at which point a proton transfer reaction occurs to yield the $\mathrm{CaCO}_{3}{ }^{0}$ (aq) ion pair. To verify this result the simulation was repeated using different initial velocities and atomic positions. Again the hydroxyl group almost instanteously diffuses away from the calcium, but this time the reaction with bicarbonate occurs after less than 300 ps. Effectively the conversion of bicarbonate to carbonate is diffusion controlled and once a hydroxide anion reaches the appropriate configuration to accept a hydrogen bond from $\mathrm{HCO}_{3}{ }^{-}$reaction takes place with negligible activation. Although it is difficult to compare this to experimental data, it is known that the equilibrium between carbonate and bicarbonate is reached in approximately $10^{-6}$ seconds $^{56}$ and so an individual proton transfer event must occur on a timescale several orders of magnitude faster than this. Our results are also in qualitative accord with previous first principles molecular dynamics that observed reaction of hydroxide with bicarbonate within $10 \mathrm{ps}$ of simulation. ${ }^{57}$ The discrepancy in time scale replicates the faster rate of proton transfer observed for the PBE functional in the microsolvation simulations and therefore may be a limitation of the exchange-correlation functional as much as the force field parameterisation.

The $\mathrm{CaHCO}_{3}{ }^{+}$(aq) ion pair is known to exist at lower $\mathrm{pH}$ and so to validate the reactive description for these conditions the above simulation was acidified by adding 
an excess proton to the above system. The starting configuration was taken as one in which the bicarbonate ion pair was still present, but the hydroxide ion had diffused to become adjacent to $\mathrm{HCO}_{3}{ }^{-}$; the extra hydrogen was then placed so as to convert the hydroxide species into a water molecule. Due to the use of a background charge the subsequent molecular dynamics run was performed in the NVT ensemble.

During the $1 \mathrm{~ns}$ of dynamics, the $\mathrm{CaHCO}_{3}{ }^{+}$(aq) ion pair is found to be stable under the acidic conditions with there being no signs of fluctuations leading to the lengthening of the $\mathrm{H}-\mathrm{O}(-\mathrm{C})$ bond. Similarly the mode of coordination of the bicarbonate to calcium remains almost unwaveringly monodentate through out the simulation; the only event of note is that the calcium switches from one carboxyl oxygen to the other after $\sim 300$ ps. The other dynamic occurrence is that the torsion angle of the hydroxyl group within the bicarbonate regularly flips between the minima about 0 and $180^{\circ}$ in a manner that is uncorrelated with the side to which the calcium ion is coordinated.

6) Basal surface of calcite.

The morphology of calcite crystals is typically dominated by a single surface termination corresponding to the (1014) surface. Consequently, most studies of calcite surface processes and growth have focused on this cleavage plane. Here we also examine this same surface in order to calibrate the quality of the present reactive model.

Before considering the more relevant situation of the (1014) surface in contact with aqueous solution, the properties of this facet in vacuo are examined. To do this, we have created a 2-D periodic slab of calcite with a depth of six layers, such that the $a b$ plane corresponds to the (211) surface of the rhombohedral setting, which is identical to the (1014) hexagonal cleavage. With conventional force fields it is traditional to use a two-region approach to the calculation of surface properties, in which the lower region is held rigid at the bulk configuration, while the upper region is allowed to relax. In this case, because of the variable charge description, it is more convenient to utilise a slab model to avoid the need to freeze the charges of the lower region at the bulk values. 
The relaxed (1014) dry surface of calcite is computed to have a surface energy of $0.445 \mathrm{~J} / \mathrm{m}^{2}$, which is in good agreement with the value of $0.39 \mathrm{~J} / \mathrm{m}^{2}$ yielded by first principles techniques using a GGA functional. ${ }^{58}$ For comparison, conventional force fields produce values anywhere from $0.23 \mathrm{~J} / \mathrm{m}^{2}$ upwards, ${ }^{59}$ with the majority offering larger surface energies in the region of $0.7 \mathrm{~J} / \mathrm{m}^{2}$. It would be expected that the use of fixed charges would lead to an overestimation of the surface energy since the reduction of ionicity at the surface is not captured. This is borne out by the atomic charges at the basal plane where the carbonate oxygens that protrude from the surface have a reduced charge of -0.78 a.u. and this is compensated by increased values of 0.98 a.u. for sub-surface oxygens. Experimentally there are some estimates of the in vacuo cleavage energies for calcite surfaces that range between $0.23 \mathrm{~J} / \mathrm{m}^{2}$ up to 0.454 $\pm 0.11 \mathrm{~J} / \mathrm{m}^{2}{ }^{60}$ However, these measurements do not correspond to the most stable basal plane.

A more controversial aspect of the basal plane of calcite is the possibility of surface reconstruction. Reports from low energy electron diffraction and atomic force microscopy indicate that the $(1014)$ surface undergoes a $(2 \times 1)$ reconstruction at low temperature and in vacuo, ${ }^{61,62}$ though it has also been proposed to exist in the presence of adsorbed molecules, such as water. Theoretically, the situation is equally unclear; while most force fields fail to yield a reconstruction, the potential of Rohl et $a l .{ }^{6}$ does indeed support the $(2 \times 1)$ structure. It is clear that the oxygen polarisability is a critical factor that influences the existence or otherwise of the reconstruction, and so naturally any rigid ion model will favour a simple surface structure. Even first principles techniques have failed to find a reconstruction thus far. Because the absolute magnitude of the imaginary mode decreases as the cell volume increases, the overestimation by many GGA functionals is sufficient to prevent the instability from existing, while LDA still does not produce an imaginary mode. It remains to be seen how more sophisticated hybrid methods fare. Based on the current reactive force field, the phonon spectrum for the relaxed $(2 \times 1)$ supercell has been computed and no imaginary mode is found, again indicating the absence of any reconstruction. Given that the underlying parameterisation of $\mathrm{C}$ and $\mathrm{O}$ was for organic species and employed information from density functional calculations it is not surprising that the degree of polarisability is not high enough to drive the instability. 
Having characterised the clean basal surface, the interaction with adsorbed water can be examined. Here there are prior calculations that examine situations from fractional coverage of a monolayer through to full simulation of the aqueous interface. For simplicity, the present model can first be benchmarked against the low coverage limit where there have been recent density functional calculations by Lardge et al. ${ }^{63}$ at the GGA level. Examination of water adsorption at this surface was also previous performed using a similar approach by Kerisit et al. ${ }^{58}$ to compute the phase diagram in the presence of $\mathrm{H}_{2} \mathrm{O}$ and $\mathrm{CO}_{2}$. However, the details of individual water configurations and binding energies were not presented in this work. Lardge et al. explored three local minima for water binding at $25 \%$ coverage with adsorption energies of $-87.8,-60.8$ and $-61.8 \mathrm{~kJ} / \mathrm{mol}$, of which the latter two are similar in nature. All configurations involve the water molecule lying approximately in a plane parallel to the surface with the oxygen coordinated to calcium at a distance of 2.39-2.41 $\AA$, while the water also forms weak hydrogen bonds to the surrounding carbonate groups.

Here water has been energy minimised on the basal surface at the same $25 \%$ coverage as Lardge et al. starting from a wide range of different positions and rotational orientations, including configurations that resemble those found from first principles methods. Starting from the configuration favoured by Lardge and coworkers, a minimum is obtained with a very similar geometry, as shown in Figure $3 a$. The Ca-O distance is in good agreement with previous calculations at $2.39 \AA$, while the hydrogen bonding distances are longer at 2.04 and $2.41 \AA$ (versus 1.97 and 2.20 $\AA$ ). This weaker hydrogen bonding strength is also reflected in the lower adsorption energy for this configuration of $-61.3 \mathrm{~kJ} / \mathrm{mol}$.

Besides the above configuration, a number of other possible binding sites for water were found. Here a greater diversity of orientations was observed in that water can also coordinate to calcium so that one $\mathrm{O}-\mathrm{H}$ bond lies parallel to the plane of the surface while the other is directed away from carbonate layer, as shown in Figure $3 \mathrm{~b}$. Despite the loss of one hydrogen bond, the adsorption energies for such states are isoenergetic with those where the water lies in the surface plane, with the most favourable configuration of this type having an adsorption energy of $-61.5 \mathrm{~kJ} / \mathrm{mol}$. It appears that the sacrifice of one hydrogen bond is compensated for by the increased strength of the other two dominant interactions. Here the Ca-O distance shortens to $2.365 \AA$, while one of the two $\mathrm{H}$ atoms has a hydrogen bond of length $2.05 \AA$. Configurations are even found in which both hydrogens are directed away from the 
surface (see Figure 3c, for example). Although they possess a less exothermic adsorption energy $(-56.7 \mathrm{~kJ} / \mathrm{mol})$, the difference is not as great as might be anticipated since some charge transfer occurs to the oxygen of water from the surface to increase the binding to calcium. Overall, the present reactive force field displays a wide range of adsorption geometries for water, all of which are quite similar in energy. As only three minima were explored in the density functional study it is not possible to assess the reliability of these alternative states. However, with the exception of the most strongly adsorbed state of Lardge et al., the adsorption energies from ReaxFF are in good agreement with those from first principles quantum mechanics. The present values also lie within the range spanned by previous values from conventional force fields $\left(-53.9,^{64}-89.1,{ }^{65}-93.9^{66} \mathrm{~kJ} / \mathrm{mol}\right)$.

Aside from physisorbed water, there is the possibility that dissociative adsorption could occur to produce a bicarbonate ion and a hydroxyl group coordinated to calcium. In their study of the non-defective basal surface, Lardge et al. found a local minimum corresponding to the chemisorbed state. However, this configuration was computed to be $171 \mathrm{~kJ} / \mathrm{mol}$ higher in energy than the most stable physisorbed state. Here we have also probed the possibility of chemisorption at $25 \%$ coverage. Three distinct chemisorbed states have been identified corresponding to the proton that forms the bicarbonate ion being in different positions relative to the hydroxyl group and it is conceivable that other minima may exist given the number of different relative torsional angles of the hydroxyl (with respect to the $\mathrm{Ca}$ and underlying surface) and bicarbonate. Energies relative to the most stable adsorbed state range from 157.7 to $189.3 \mathrm{~kJ} / \mathrm{mol}$, thus agreeing with the first principles results in indicating that water should not dissociate on the non-defective (1014) calcite surface. While the exothermicity of chemisorption is slightly underestimated with respect to the GGA result, the majority of this discrepancy is due to the most stable adsorbed state being under bound, rather than any error in the energy of the chemisorbed configuration.

So far the adsorption of water at low coverage has been considered. However, more interesting is the aqueous-calcite interface for liquid water. To simulate this we have built a 4 × 3 supercell of the calcite slab containing 960 atoms and placed a layer of 256 water molecules above the surface within periodic boundary conditions. The approximate dimensions of this cell are $20 \times 24.5$ x $40 \AA$. Molecular dynamics was then performed within the NPT ensemble with the cell constrained to remain 
orthorhombic. After equilibration, the structure of the water immediately above the surface remains remarkable constant through out the production phase. Here a regular array of water molecules is found with the oxygen coordinated to the calcium of the underlying surface, while the hydrogens interact primarily with water molecules from the liquid (Figure 4). Each individual molecule of this ordered layer resembles the orientation found for an isolated water in the least stable of the three configurations found at $25 \%$ coverage (see Figure $3 \mathrm{c}$ ). The fact that the system prefers to adopt this configuration as the basis for the first layer of water can be readily understood since the extra stability arising from water-water hydrogen bonds easily out weighs the 5 $\mathrm{kJ} / \mathrm{mol}$ penalty for reduced hydrogen bonding to the carbonate groups of the calcite.

The structure of the aqueous-calcite interface has been studied both experimentally, by surface X-ray scattering, ${ }^{3}$ and theoretically, by molecular dynamics based on traditional potentials. Experimentally, water is found to form two distinct layers of water at heights of $2.3 \pm 0.1$ and $3.5 \pm 0.2 \AA$ above the first calcium carbonate plane. The water also exhibits lateral ordering with respect to the underlying ions, with the first water being displaced relative to calcium. The most recent simulation study, which employed a force field that had been accurately calibrated against the thermodynamics and structural properties of the calcium and carbonate ions in solution, also supports the idea of two ordered water layers, with the possibility of perhaps a weakly ordered third layer. ${ }^{7}$ Where previous theory and experiment disagree is in the height of the water layers, with most simulations suggesting that the perpendicular height of the first water above the surface should be closer to $2 \AA$, with the second layer also correspondingly closer.

In our previous unreactive model, ${ }^{7}$ the first layer of water has one O-H group in the plane parallel to the surface, while the second $\mathrm{O}-\mathrm{H}$ is hydrogen bonded to other water molecules, closer to the configuration of Figure 3b. Given the subtle energetic differences between the minima involved it is difficult to be absolutely certain which force field is more accurate. The present reactive model yields an average perpendicular distance from $\mathrm{Ca}$ to the water layer of $\sim 2.3 \AA$, closer to experiment than previous force fields, except that of Perry IV et al., ${ }^{67}$ but as a consequence disagrees with experiment in suggesting that the water oscillates about a site directly above calcium. These conflicting observations are caused by the fact that all force fields give similar Ca-O bond lengths and so only the angle of this vector relative to the surface normal changes. If it is argued that the vertical resolution of the X-ray 
scattering is more certain than the lateral one, then the present model would be more accurate, while the converse situation would favour other force fields. Currently there appears to be no unambiguous resolution of this debate, though the proposal of a different orientation for the first ordered water layer provides an alternative that is consistent with different aspects of the X-ray data.

For the second layer of water, a common orientation is found between the present work and our previous simulations. Here the water molecule has an $\mathrm{O}-\mathrm{H}$ bond parallel to the surface normal such that it can hydrogen bond to the underlying carbonate anions through the first strongly ordered water layer. Structurally, this layer of water is much less well defined in the present simulations and water molecules are observed to fluctuate between this state and other orientations on the timescale accessible.

The issue of speciation at the surface of calcite has attracted considerable attention, ${ }^{68,69,70}$ especially with regard to the bicarbonate-carbonate equilibrium, the deprotonation of water coordinated to calcium and the level of adsorbed ions. In our reactive simulations, no protonation of carbonate or deprotonation of the first ordered water layer are observed on the timescale of the simulation. While more extensive studies are required to determine the free energy landscape and draw an absolute conclusion, the present findings are consistent with the statement by van Cappellen et $a l^{71}$ that "in the $\mathrm{pH}$ range 5.5 to 8 the surface is made of nearly constant and equal amounts of $>\mathrm{CaOH}_{2}{ }^{+}$and $>\mathrm{CO}_{3}{ }^{2-}$ sites". This also validates our prior unreactive simulations where no dissociation was possible. Here free energy profiles indicate that adsorbed species, especially $\mathrm{Ca}^{2+}$, will prefer to be solvent separated from the underlying surface rather than directly coordinated.

\section{Conclusions}

A new reactive force field for aqueous-calcium carbonate systems has been derived based on the ReaxFF methodology. Unlike previous parameterisations, the simplifying approximation is made that calcium is a fixed charge di-cation that interacts by screened electrostatics of the usual ReaxFF form and short-range repulsive potentials. Whilst this approximation limits the applicability of the current model to calcium in ionic environments, this simple approach appears to be satisfactory for describing much of the chemistry of calcium carbonate in contact with 
water where the main reactivity involves the equilibria between carbonic acid, bicarbonate and carbonate. Reactions involving dissolved carbon dioxide in water can also be described, though the energetics of conversion between two and threecoordinate carbon are less satisfactory than proton transfer reactions.

Calculations have been performed to examine the speciation of carbonate in different environments spanning only 8 water molecules, through microsolvation in a droplet of 100 waters, to bulk solvation in periodic boundary conditions. As the level of hydration increases, the stability of the carbonate and bicarbonate anions increases, as would be expected, and the lifetime of $\mathrm{CO}_{3}{ }^{2-}$ increases. Up to the size of the droplet considered, carbonic acid is ultimately produced in neutral conditions; a finding that is common to both first principles and ReaxFF based molecular dynamics. Because of the longer timescale that can be accessed, the reactive force field is able to observe the diffusion to the surface of the droplet indicating that it prefers the hydrophobic environment of the interface for the $\mathrm{C}=\mathrm{O}$ group while allowing the hydroxyls to remain hydrogen bonded. In all simulations the protonation of bicarbonate occurs to yield the metastable cis-trans isomer of carbonic acid due to the alignment of the incoming proton with the dipole moment associated with the hydrogen position in $\mathrm{HCO}_{3}{ }^{-}$. Once in bulk solution then both carbonate and bicarbonate are stable as local minima for the duration of all simulations.

Although it is not possible to explore a wide range of $\mathrm{pH}$ conditions, due to the limitations of simulation size, the present work demonstrates that for neutral conditions the bicarbonate anion becomes unstable with respect to carbonate when complexed in an ion pair. Given that calcium carbonate is typically grown under basic conditions, this suggests that models that invoke the carbonate anion as the growth species are valid once an ion pair is formed. Importantly, this indicates that conclusions drawn from conventional force fields, where $\mathrm{CO}_{3}{ }^{2-}$ has to be assumed to be involved even under conditions where it is not the dominant equilibrium species in solution, are highly likely to be valid (subject to the quality of other aspects of the parameterisation). For the $\mathrm{CaCO}_{3}{ }^{0}$ (aq) ion pair, the monodentate binding of calcium to carbonate is found to be more stable with the bidentate state having a transitory existence during the exchange of the coordinating oxygen. Similarly, the $\mathrm{CaHCO}_{3}{ }^{+}{ }_{\text {aq }}$ ion pair also favours monodentate binding.

Results obtained with the reactive force field are generally in good agreement with those from experiment, where available, and first principles quantum mechanical 
calculations. Given that the ReaxFF approach is orders of magnitude faster than density functional methods, this should allow more extensive probing of carbonate chemistry in solution in future than would otherwise be possible. Furthermore, although the use of ReaxFF requires recalculation of charges at every configuration, the short-range nature of the Coulomb interaction means that this reactive force field is faster to compute in GULP (by approximately a factor of 5) than a conventional flexible model for large systems where domain decomposition is possible. Since the rate of water exchange for calcium ions occurs on a timescale of the order of $100 \mathrm{ps,}$ the use of nanosecond simulations must be considered mandatory for molecular dynamics in this field to ensure that the configurations are even locally sampled, and this is now feasible with the inclusion of reactivity.

\section{Acknowledgements}

JDG and PR thank the Australian Research Council for research fellowships and support through the Discovery Program, as well as iVEC and NCI for provision of computing resources. 
Table 1 Fitted parameters for the interaction of calcium with oxygen, hydrogen and carbon derived in the present study. Here the $\mathrm{Ca}-\mathrm{H}$ and $\mathrm{Ca}-\mathrm{C}$ interactions are represented using a Buckingham potential (Buck), while the $\mathrm{Ca}-\mathrm{O}$ contribution is fitted with a Lennard-Jones (LJ) 12-6 potential. In both cases only the repulsive term is included and the $\mathrm{C}_{6}$ coefficient is set to zero. All repulsive potentials are cut-off at $10 \AA$. The representation of parameters to significant numbers of decimal places is purely to allow precise reproduction of published quantities and does not imply accuracy at this level.

\begin{tabular}{|l|c|c|c|c|}
\hline Interaction & $\gamma\left(\AA^{-1}\right)$ & $\mathrm{A}_{\mathrm{LJ}}\left(\mathrm{eV} \AA^{12}\right)$ & $\mathrm{A}_{\text {Buck }}(\mathrm{eV})$ & $\rho_{\text {Buck }}(\AA)$ \\
\hline $\mathrm{Ca}$ Coulomb & 0.6 & - & - & - \\
\hline $\mathrm{Ca}-\mathrm{O}$ & - & 4700.0 & - & - \\
\hline $\mathrm{Ca}-\mathrm{H}$ & - & - & 310.47299 & 0.337744 \\
\hline $\mathrm{Ca}-\mathrm{C}$ & - & - & 723.40055 & 0.333801 \\
\hline
\end{tabular}


Table 2 Comparison of experimental and calculated structure and properties for solid phases containing calcium used as part of the training set for fitting. NB the values for aragonite are quoted after removal of 3 imaginary modes.

\begin{tabular}{|c|c|c|c|}
\hline Structure/property & Experiment & Calculated & Error $(\%)$ \\
\hline Calcite, $a(\AA)^{23}$ & 4.988 & 4.903 & 1.70 \\
\hline Calcite, $c(\AA)$ & 17.061 & 17.153 & 0.54 \\
\hline $\begin{array}{l}\text { Calcite, bulk modulus } \\
(\mathrm{GPa})^{31}\end{array}$ & $73.46 \pm 0.27$ & 73.7 & 0.33 \\
\hline Calcite, $\mathrm{r}(\mathrm{C}-\mathrm{O})(\AA)$ & 1.284 & 1.283 & 0.08 \\
\hline Aragonite, $a(\AA)^{72}$ & 5.740 & 6.117 & 6.57 \\
\hline Aragonite, $b(\AA)$ & 4.961 & 4.837 & 2.50 \\
\hline Aragonite, $c(\AA)$ & 7.967 & 8.379 & 4.92 \\
\hline $\begin{array}{l}\text { Portlandite, } a(\AA) \text { at } 0.07 \\
\mathrm{GPa}^{24}\end{array}$ & 3.5717 & 3.4063 & 4.63 \\
\hline $\begin{array}{l}\text { Portlandite, } c(\AA) \text { at } 0.07 \\
\text { GPa }\end{array}$ & 4.8743 & 5.2109 & 6.91 \\
\hline Calcium oxide, $a(\AA)$ & 4.8075 & 4.3586 & 9.34 \\
\hline Calcium carbide, $a(\AA)^{21}$ & 3.8863 & 3.7590 & 3.28 \\
\hline Calcium carbide, $c(\AA)$ & 6.3862 & 6.6095 & 3.38 \\
\hline $\begin{array}{l}\text { Calcium hydride, } a(\AA) \\
22\end{array}$ & 5.9285 & 5.7088 & 3.71 \\
\hline Calcium hydride, $b(\AA)$ & 3.5777 & 3.5746 & 0.09 \\
\hline Calcium hydride, $c(\AA)$ & 6.7896 & 6.9279 & 2.04 \\
\hline
\end{tabular}


Table 3 Atomic charges for $\mathrm{C}, \mathrm{O}$ and $\mathrm{H}$ for solid-state materials.

\begin{tabular}{|l|c|c|}
\hline Compound/phase & Atom & Charge (a.u.) \\
\hline Calcite & C & +0.7906 \\
\hline & O & -0.9302 \\
\hline Aragonite & C & +0.7978 \\
\hline & O & -0.9326 \\
\hline Portlandite & O & -1.2555 \\
\hline & H & +0.2555 \\
\hline
\end{tabular}


Table 4: Reaction energies $(\mathrm{kJ} / \mathrm{mol})$ for water molecules binding to a calcium dication in vacuo for $\mathrm{Ca}\left(\mathrm{H}_{2} \mathrm{O}\right)_{\mathrm{n}-1}{ }^{2+}+\mathrm{H}_{2} \mathrm{O} \rightarrow \mathrm{Ca}\left(\mathrm{H}_{2} \mathrm{O}\right)_{\mathrm{n}}{ }^{2+}$. Literature values are quoted from the density functional study of Bakó et al. ${ }^{41}$ and shell model of Kerisit and Parker. ${ }^{73}$ Hydration energies are also included for the recent unreactive flexible force field of Raiteri and Gale. ${ }^{8}$

\begin{tabular}{|c|c|c|c|c|}
\hline $\mathrm{n}$ & $\begin{array}{c}\mathrm{BLYP} / \\
6-311+\mathrm{G}^{* *}\end{array}$ & Shell model & $\begin{array}{c}\text { Flexible } \\
\text { Unreactive }\end{array}$ & $\begin{array}{c}\text { Reactive } \\
\text { (This work) }\end{array}$ \\
\hline 1 & -238.9 & -153.0 & -208.3 & -225.8 \\
\hline 2 & -200.5 & -147.9 & -201.3 & -208.3 \\
\hline 3 & -188.4 & -139.1 & -187.5 & -185.0 \\
\hline 4 & -151.1 & -130.5 & -174.2 & -165.0 \\
\hline 5 & -110.5 & -116.1 & -149.8 & -120.0 \\
\hline 6 & -103.2 & -110.9 & -139.7 & -111.5 \\
\hline 7 & -55.1 & -73.3 & -80.9 & -48.0 \\
\hline Sum $1 \rightarrow 6$ & -992.6 & -797.5 & -1060.8 & -1063.6 \\
\hline Sum $1 \rightarrow 7$ & -1047.7 & -870.8 & -1141.7 & -1111.6 \\
\hline
\end{tabular}


Table 5 Total energy differences $(\mathrm{kJ} / \mathrm{mol})$ for dissociation reactions of the trans-trans conformation of carbonic acid.

\begin{tabular}{|c|c|c|}
\hline Reaction & M06-L/aug-cc-pvtz & ReaxFF \\
\hline $\mathrm{H}_{2} \mathrm{CO}_{3(\mathrm{~g})} \rightarrow \mathrm{HCO}_{3}^{-}(\mathrm{g})+\mathrm{H}_{(\mathrm{g})}^{+}$ & +1458 & +1341 \\
\hline $\mathrm{H}_{2} \mathrm{CO}_{3(\mathrm{~g})}+\mathrm{H}_{2} \mathrm{O}_{(\mathrm{g})} \rightarrow \mathrm{HCO}_{3}^{-}{ }_{(\mathrm{g})}+\mathrm{H}_{3} \mathrm{O}_{(\mathrm{g})}^{+}$ & +731 & +784 \\
\hline $\mathrm{H}_{2} \mathrm{CO}_{3(\mathrm{~g})}+2 \mathrm{H}_{2} \mathrm{O}_{(\mathrm{g})} \rightarrow \mathrm{HCO}_{3}^{-}(\mathrm{g})+\mathrm{H}_{5} \mathrm{O}_{2}^{+}(\mathrm{g})$ & +587 & +588 \\
\hline
\end{tabular}


Figure 1 Configurations taken from the molecular dynamics of $2\left(\mathrm{H}_{3} \mathrm{O}^{+}\right) \cdot\left(\mathrm{CO}_{3}{ }^{2-}\right.$ ). $6 \mathrm{H}_{2} \mathrm{O}$, a) Initial configuration, b) configuration after $10 \mathrm{ps}$ at $200 \mathrm{~K}$, and c) configuration just following the second proton transfer after 1.78 ps at $300 \mathrm{~K}$. Carbon, oxygen and hydrogen are coloured green, red and white, respectively, while dashed lines represent hydrogen bonds.

a)

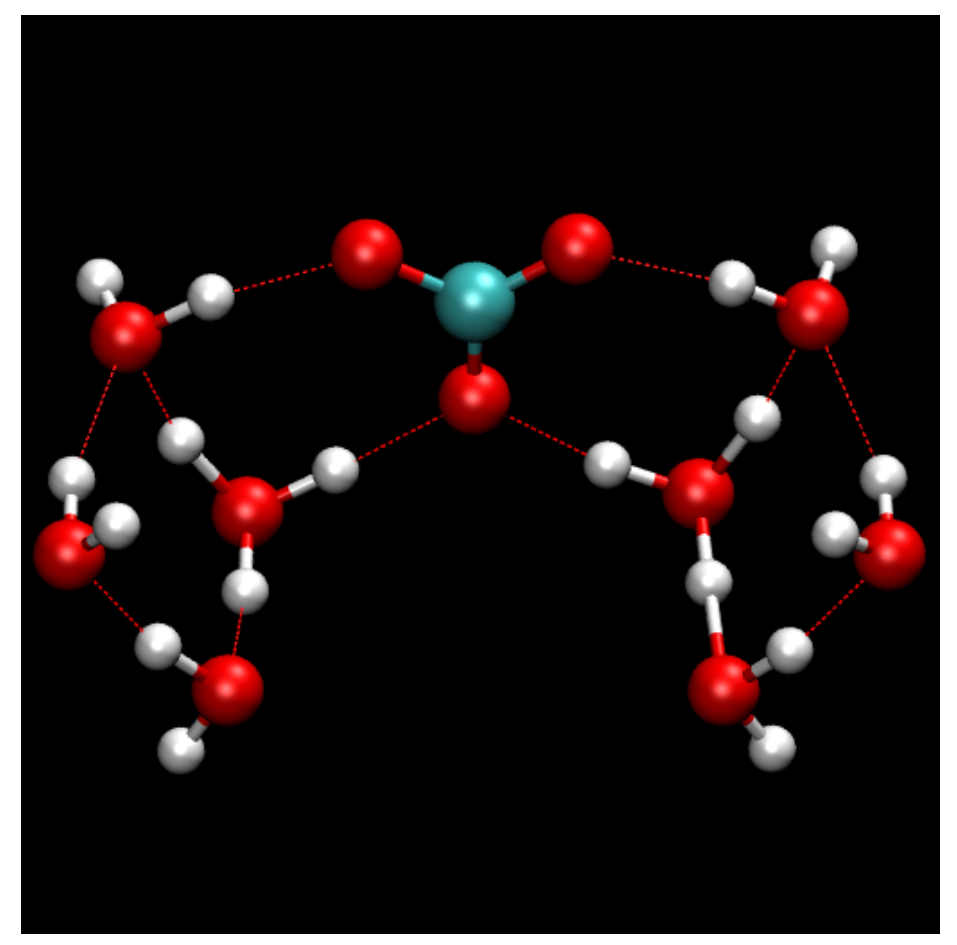


Figure 1 continued

b)

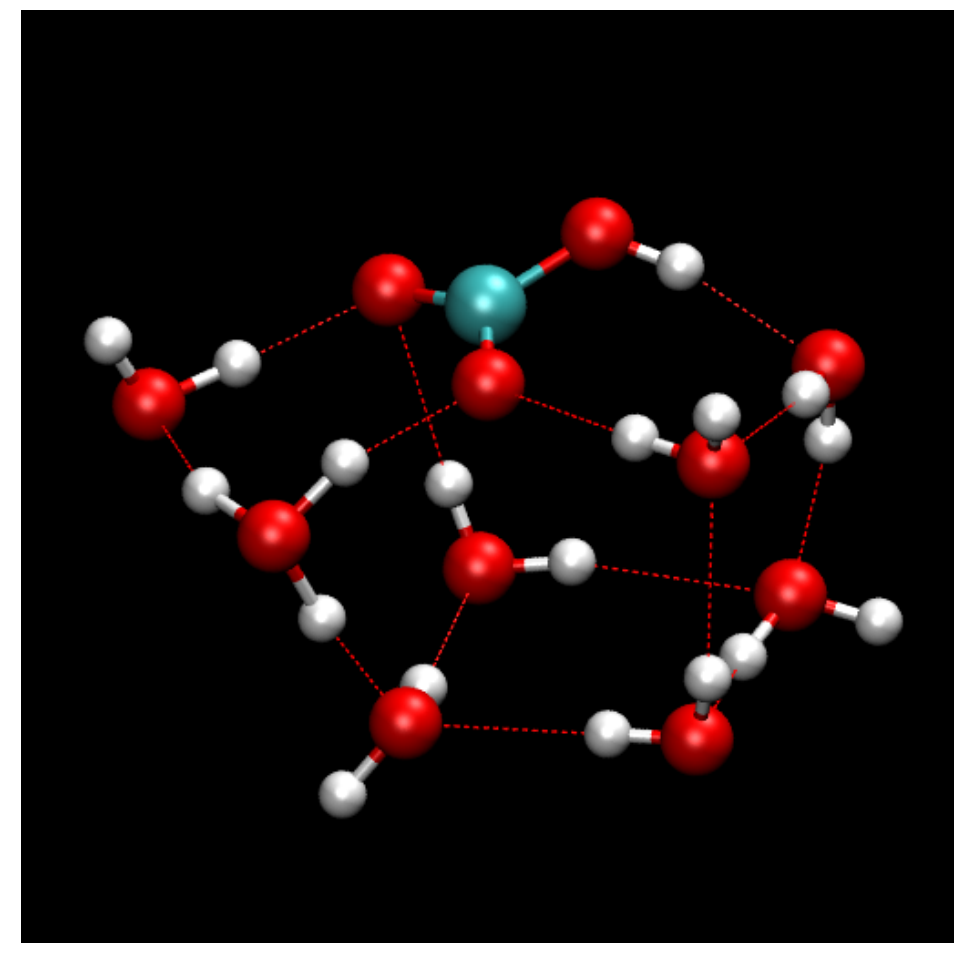


Figure 1 continued

c)

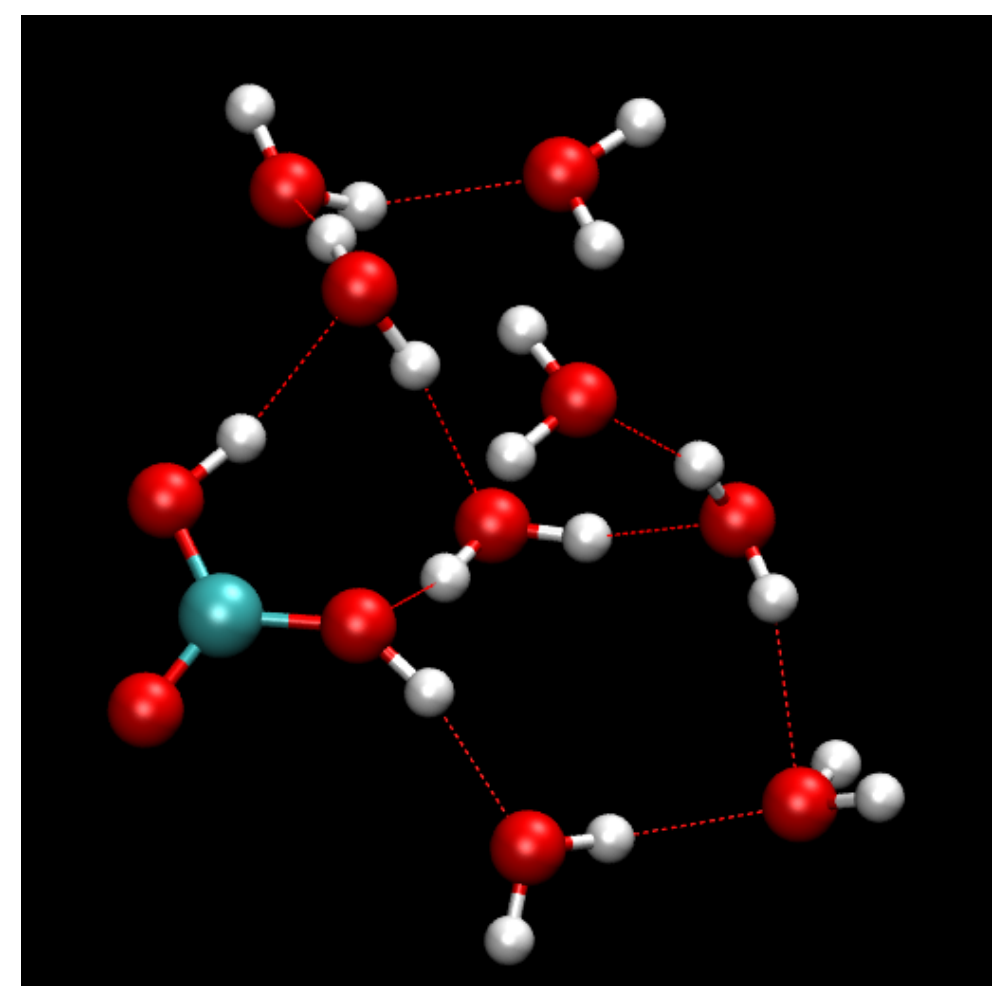


Figure 2 Configuration taken from the molecular dynamics of a carbonate anion in a droplet containing 98 water molecules and 2 hydronium ions highlighting the Grotthuss proton transfer event that leads to the formation of bicarbonate. Configuration shown is taken from the first principles dynamics, but the same mechanism is observed in the trajectory of the reactive force field.

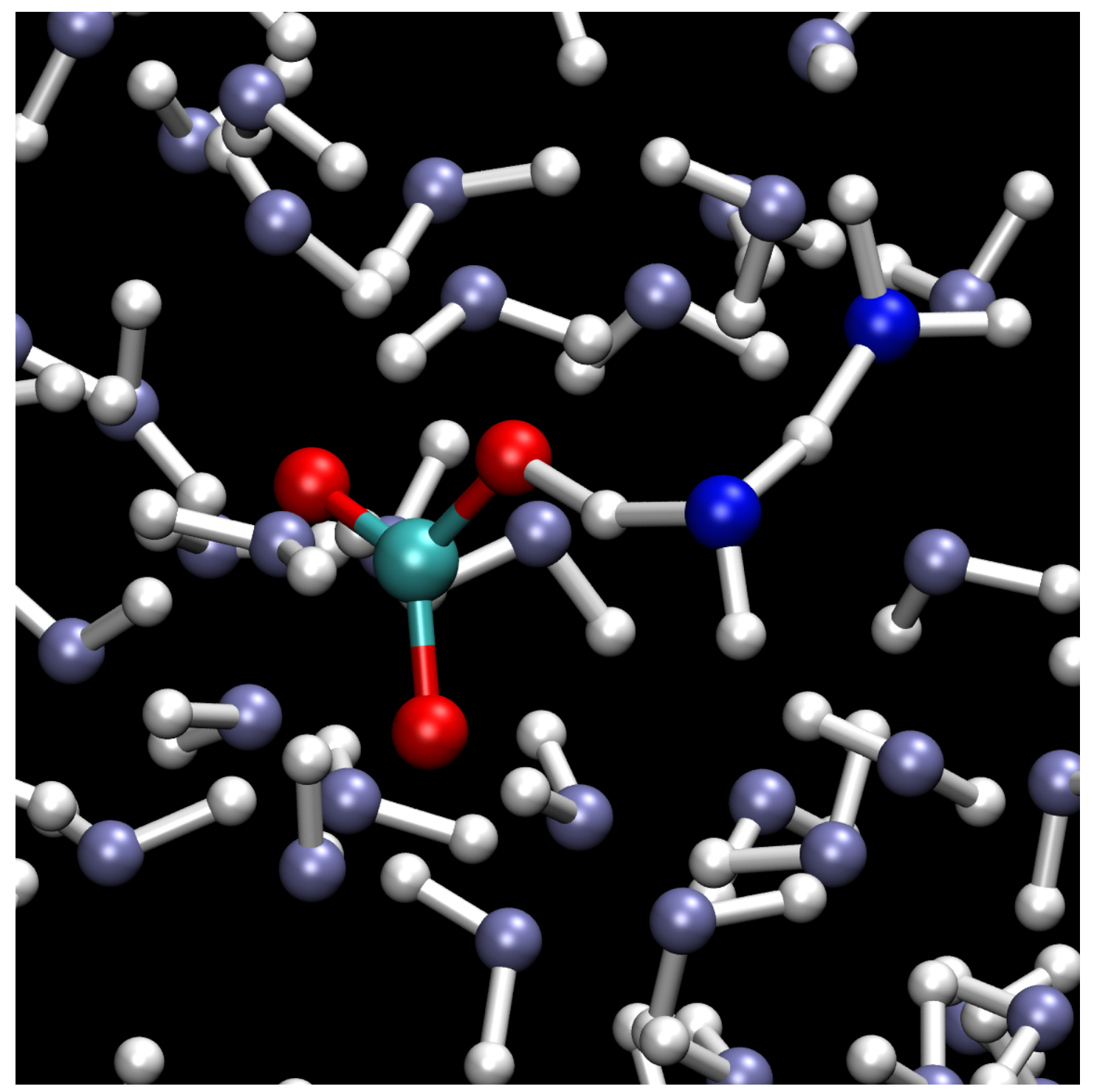


Figure 3: Three distinct types of energy minimised configurations for water on the basal surface of calcite at $25 \%$ coverage. a) Two hydrogen bonds; the lowest energy structure found by Lardge et $a l .{ }^{63}$ (see Figure $2 \mathrm{~b}$ in their paper), b) one hydrogen bond and c) no hydrogen bonds to the calcite surface.

a)

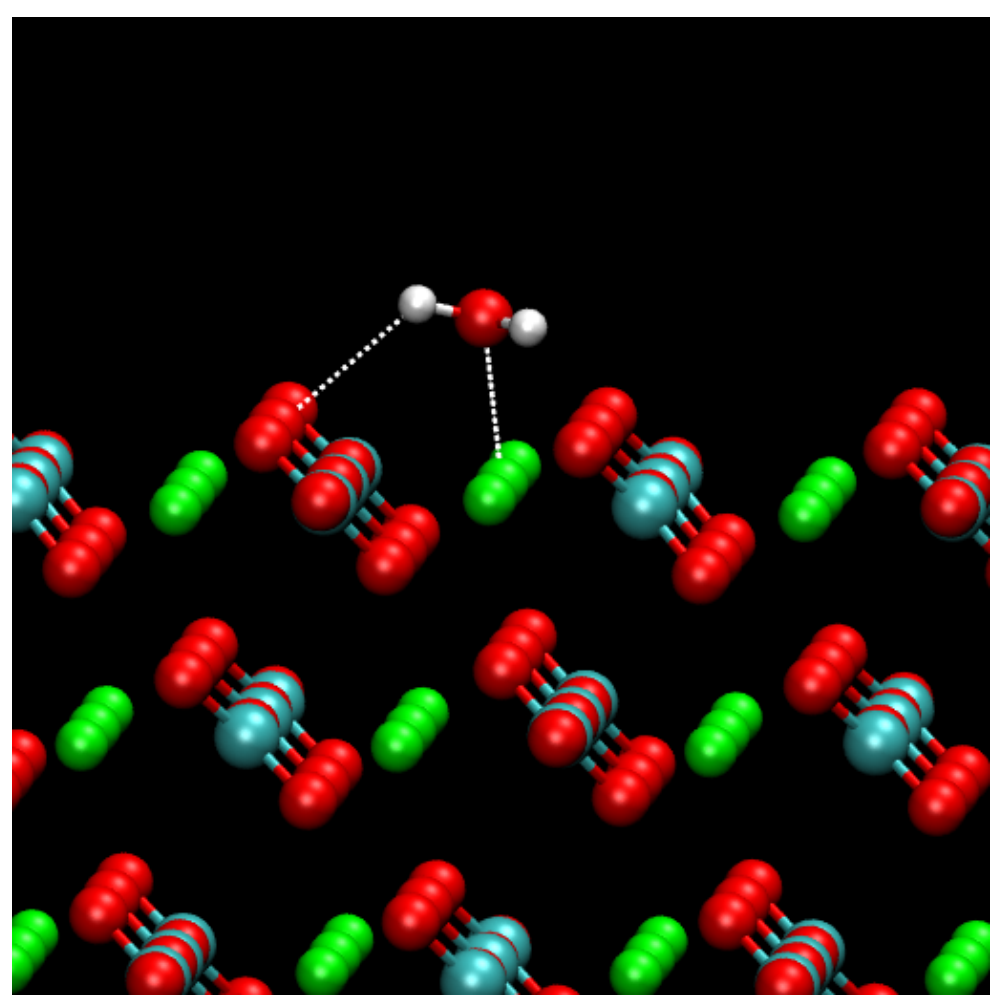


Figure 3 continued

b)

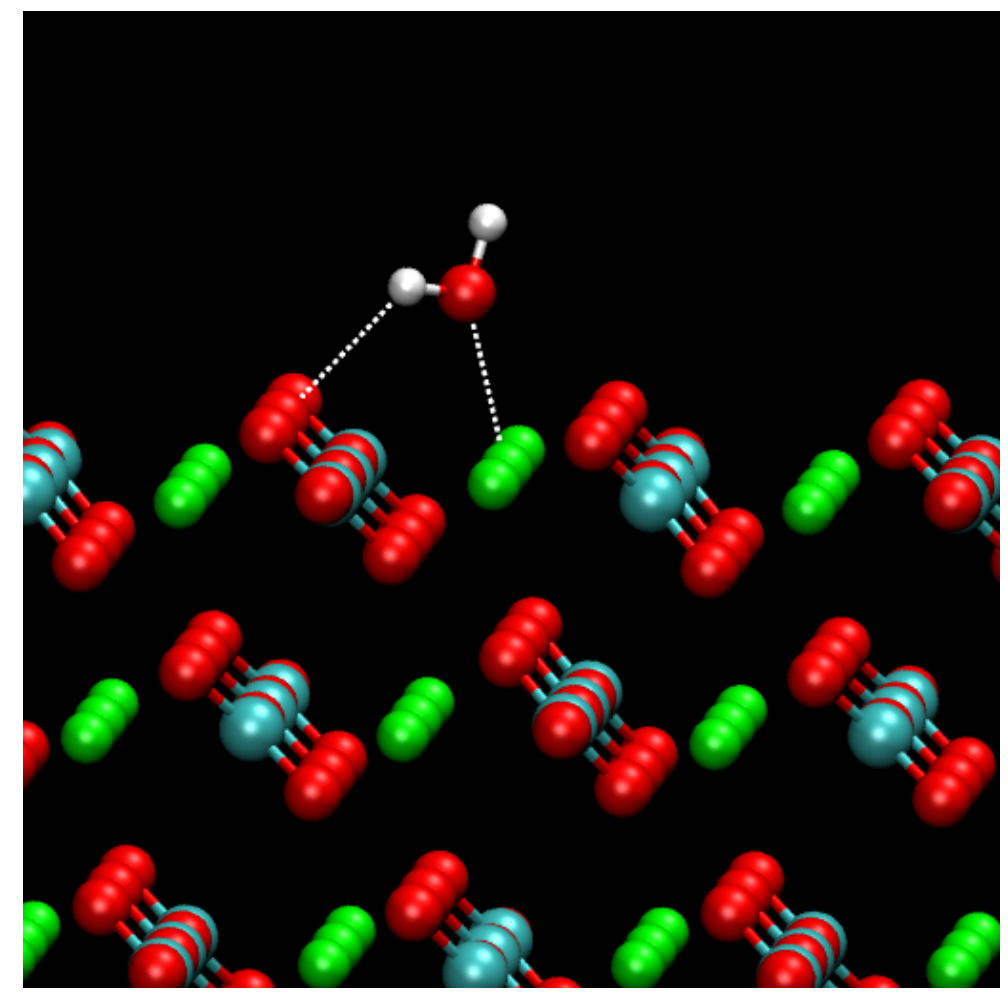


Figure 3 continued

c)

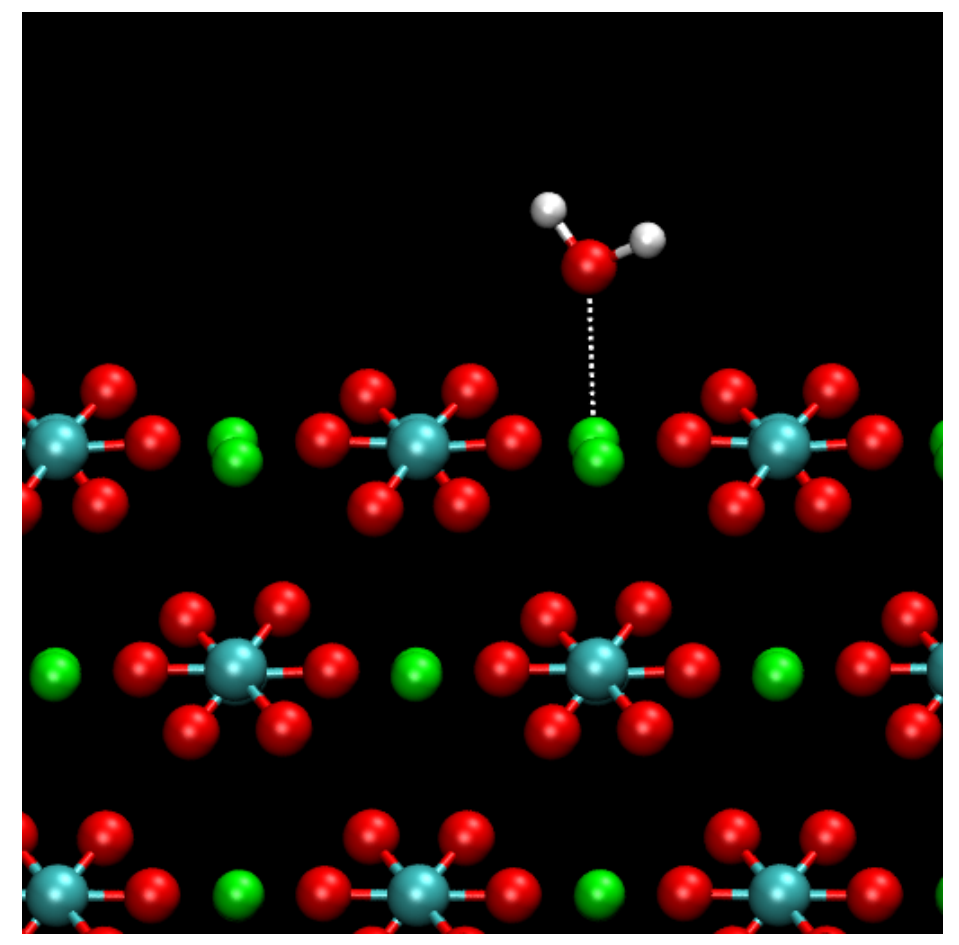


Figure 4: An instantaneous configuration of the basal surface of calcite in contact with water taken from the molecular dynamics simulation of the interface as shown from two directions.

a)

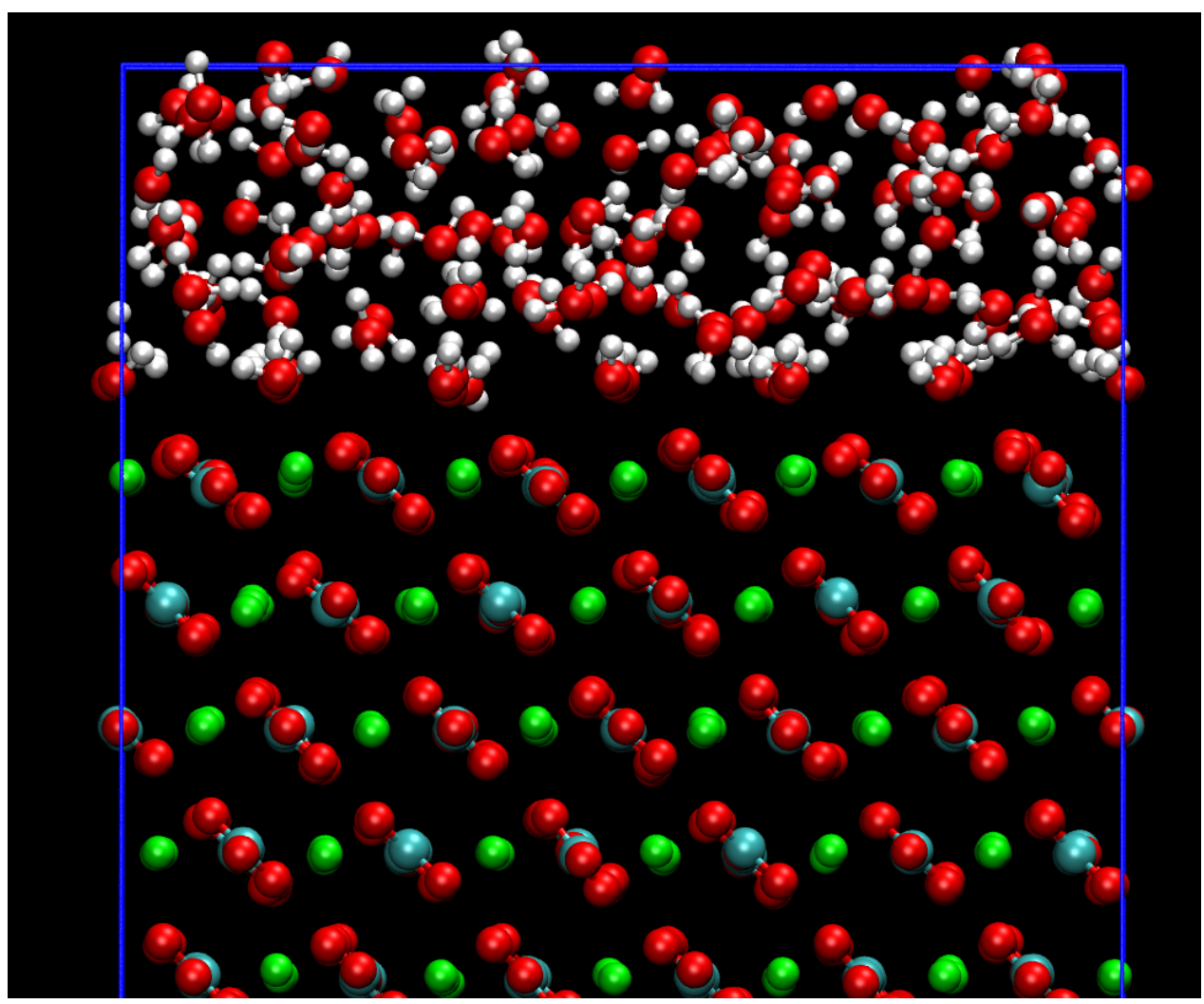


Figure 4 continued

b)

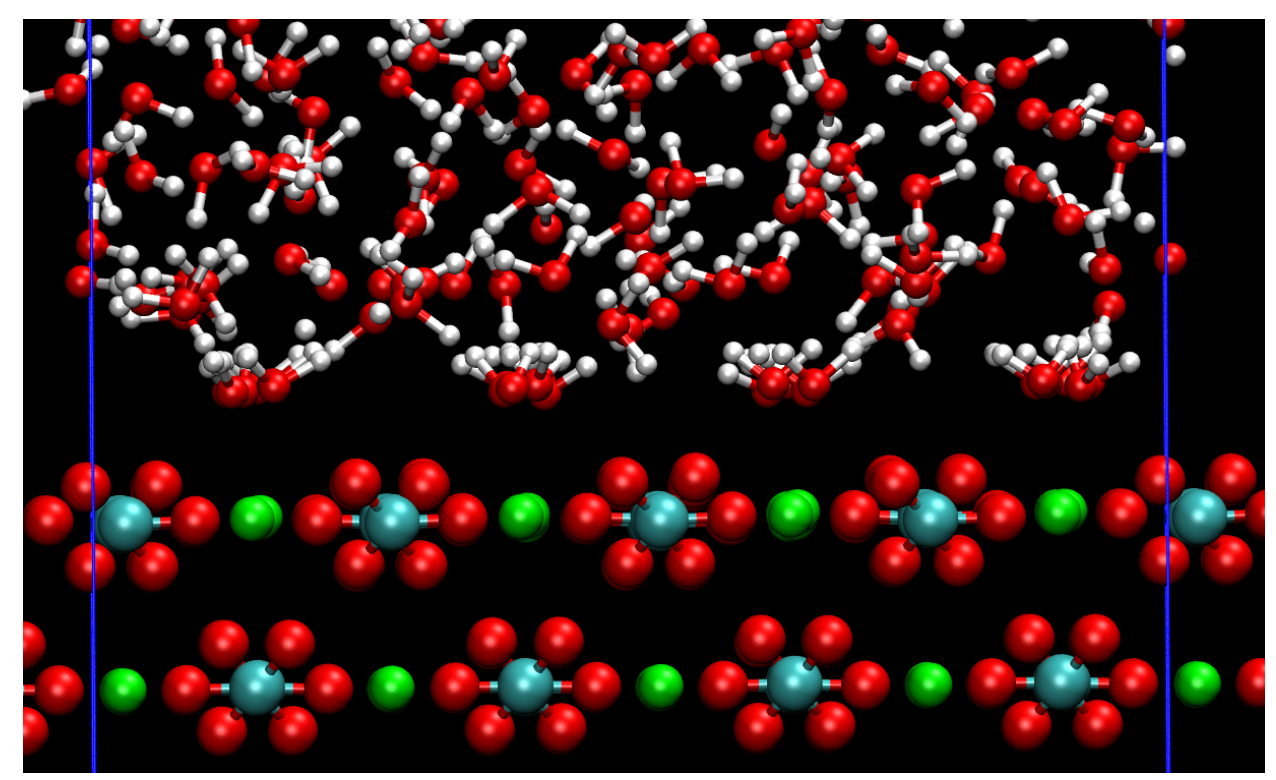




\section{References}

${ }^{1}$ D. Gebauer, A. Völkel and H. Cölfen, Science, 2008, 322, 1819-1822.

${ }^{2}$ A.J. Gratz, P.E. Hillner and P.K. Hansma, Geochim. Chim. Acta, 1993, 57, 491-495.

${ }^{3}$ P. Geissbühler, P. Fenter, E. DiMasi, G. Srajer, L.B. Sorensen and N.C. Sturchio, Surf. Sci., 2004, 573, 191-203.

${ }^{4}$ S. Elhadj, J.J. de Yoreo, J.R. Hoyer and P. Dove, Proc. Natl. Acad. Sci. USA, 2006, 103, 19237-19242.

${ }^{5}$ A. Pavese, M. Catti, S.C. Parker and A. Wall, Phys. Chem. Miner., 1996, 23, 89-93.

${ }^{6}$ A.L. Rohl, K. Wright and J.D. Gale, Am. Miner., 2003, 88, 921-925.

${ }^{7}$ P. Raiteri, J.D. Gale, D. Quigley and P.M. Rodger, J. Phys. Chem. C, 2010, 114, 5997-6010.

${ }^{8}$ P. Raiteri and J.D. Gale, J. Am. Chem. Soc., 2010, 132, 17623-17634.

${ }^{9}$ J. Wang, G. Román-Pérez, J.M. Soler, E. Artacho and M.-V. Fernández-Serra, J. Chem. Phys., 2011, 134, 024516.

${ }^{10}$ Y. Wu, H. Chen, F. Wang, F. Paesani and G.A. Voth, J. Phys. Chem. B, 2008, 112, 467-482.

${ }^{11}$ C.D. Wick and L.X. Dang, J. Phys. Chem. A, 2009, 113, 6356-6364.

${ }^{12}$ P. Raiteri, J.D. Gale and G. Bussi, J. Phys. Cond. Matter, 2011, 23, 334213.

${ }^{13}$ J.H. Los, L.M. Ghiringhelli, E.J. Meijer and A. Fasolino, Phys. Rev. B, 2005, 72, 214102.

${ }^{14}$ A.C.T. van Duin, S. Dasgupta, F. Lorant and W.A. Goddard III, J. Phys. Chem. A, 2001, 105, 9396-9409.

${ }^{15}$ W.J. Mortier, S.K. Ghosh and S. Shankar, J. Am. Chem. Soc., 1986, 108, 43154320.

${ }^{16}$ K. Chenoweth, A.C.T. van Duin and W.A. Goddard III, J. Phys. Chem. A, 2008, 112, 1040-1053.

${ }^{17}$ J.D. Gale and A.L. Rohl, Mol. Simul., 2003, 29, 291-341.

${ }^{18}$ J.D. Gale, Z. Krist., 2005, 220, 552-554.

${ }^{19}$ D. Raymond, A.C.T. van Duin, M. Baudin and K. Hermansson, Surf. Sci., 602, 1020-1031.

${ }^{20}$ S. Cheung, W.-Q. Deng, A.C.T. van Duin and W.A. Goddard III, J. Phys. Chem. A, 2005, 109, 851-859.

${ }^{21}$ O. Reckeweg, A. Baumann, A. Mayer, J. Glaser and H.-J. Meyer, Z. Anorg. Allg. Chem., 1999, 625, 1686-1692.

${ }^{22}$ H. Wu, W. Zhou, T.J. Udovic, J.J. Rush and T. Yildirim, J. Alloy Comp., 2007, 436, 51-55.

${ }^{23}$ S.A. Markgraf and R. Reeder, Am. Miner., 1971, 70, 590-600.

${ }^{24}$ H.W. Xu, Y.S. Zhao, J.Z. Zhang, D.D. Hickmott and L.L. Daemen, Phys. Chem. Miner., 2007, 34, 223-232.

25 J. Chen and T.J. Martinez, Chem. Phys. Lett., 2007, 438, 315-320.

${ }^{26}$ Y. Zhao and D.G. Truhlar, J. Chem. Phys., 2006, 125, 194101.

${ }^{27}$ M. Valiev, E.J. Bylaska, N. Govind, K. Kowalski, T.P. Straatsma, H.J.J. van Dam, D. Wang, J. Nieplocha, E. Apra, T.L. Windus and W.A. de Jong, Comput. Phys.

Commun., 2010, 181, 1477-1489.

${ }^{28}$ J.M. Soler, E. Artacho, J.D. Gale, A. García, J. Junquera, P. Ordejón and D.

Sánchez-Portal, J. Phys. Cond. Matter, 2002, 14, 2745-2779.

${ }^{29}$ J.P. Perdew, K. Burke and M. Ernzerhof, Phys. Rev. Lett., 1996, 77, 3865-3868.

${ }^{30}$ N. Troullier and J.L. Martins, Phys. Rev. B, 1991, 43, 1993-2006.

${ }^{31}$ S.A.T. Redfern and R.J. Angel, Contrib. Miner. Petrol., 1999, 134, 102-106. 
${ }^{32}$ C.C. Chen, C.C. Lin, L.G. Liu, S.V. Sinogelkin and J.D. Bass, Am. Miner., 2001, 86, 1525-1529.

${ }^{33}$ C.L. Freeman, J.H. Harding, D.J. Cooke, J.A. Elliot, J.S. Lardge and D.M. Duffy, J. Phys. Chem. C, 2007, 111, 11943-11951.

${ }^{34}$ G. Wolf, J. Lerchner, H.G. Schmidt, H. Gamsjager, E. Konigsberger and P. Schmidt, J. Therm. Anal., 1996, 46, 353-359.

${ }^{35}$ A.C.T. van Duin, B.V. Merinov, S.S. Han, C.O. Dorso and W.A. Goddard III, J. Phys. Chem. A, 2008, 45, 11414-11422.

${ }^{36}$ S. Peziale, H.J. Reichmann, F.R. Schilling, H.R. Wenk and P.J.M. Monteiro, Cement Concrete Res., 2008, 38, 1148-1153.

${ }^{37}$ P. Ugliengo, C.M. Zicovich-Wilson, S. Tosoni and B. Civalleri, J. Mater. Chem., 2009, 19, 2563-2572.

${ }^{38} \mathrm{Ph}$. Baranek, A. Lichanot, R. Orlando and R. Dovesi, Chem. Phys. Lett., 2001, 340, 362-369.

${ }^{39}$ T. Megyes, T. Grosz, T. Radnai, I. Bako and G. Palinkas, J. Phys. Chem. A, 2004, 108, 7261-7271.

${ }^{40}$ H. Ohtaki and T. Radnai, Chem. Rev., 1993, 93, 1157-1204.

${ }^{41}$ L. Bakó, J. Hutter and G. Palinkas, J. Chem. Phys., 2002, 117, 9838-9843.

${ }^{42}$ A.C.T. van Duin, V.S. Bryantsev, M.S. Diallo, W.A. Goddard III, O. Rahaman, D.J. Doren, D. Raymand and K. Hermansson, J. Phys. Chem. A, 2010, 114, 95079514

${ }^{43}$ J.R. Rustad, S.L. Nelmes, V.E. Jackson and D.A. Dixon, J. Phys. Chem. A, 2008, 112, 542-555.

${ }^{44}$ C.A. Wight and A.L. Boldyrev, J. Phys. Chem., 1995, 99, 12125-12130.

${ }^{45}$ K.R. Liedl, S. Sekusak and E. Mayer, J. Am. Chem. Soc., 1997, 119, 3782-3784.

${ }^{46}$ X. Huang, B.J. Braams and J.M. Bowman, J. Chem. Phys., 2005, 122, 044308.

${ }^{47}$ H.-S. Andrei, S.A. Nizkorodov and O. Dopfer, Angew. Chem. Int. Ed., 2007, 46, 4754-4756.

${ }^{48}$ X. Wang, W. Conway, R. Burns, N. McCann and M. Maeder, J. Phys. Chem. A, 2010, 114, 1734-1740.

${ }^{49}$ J.R. Rustad, S.L. Nelmes, V.E. Jackson and D.A. Dixon, J. Phys. Chem. A, 2008, 112, 542-555.

${ }^{50}$ A. Stirling and I. Pápai, J. Phys. Chem. B, 2010, 114, 16854-16859.

${ }^{51}$ W. Hage, K.R. Liedl, A. Hallbrucker and E. Mayer, Science, 1998, 279, 1332-1335.

${ }^{52}$ V. Tognetti and C. Adamo, J. Phys. Chem. A, 2009, 113, 14415-14419.

${ }^{53}$ P. Kumar, A. Kalinichev and R. Kirkpatrick, J. Phys. Chem. B, 2009, 113, 794-802.

${ }^{54}$ A. Gray-Weale and J.K. Beattie, Phys. Chem. Chem. Phys., 2009, 11, 10994-11005.

${ }^{55}$ G.A. Tribello, F. Bruneval, C.C. Liew and M. Parrinello, J. Phys. Chem. B, 2009, 113, 11680-11687.

${ }^{56}$ A.R. Davis and B.G. Oliver, J. Solution Chem., 1972, 1, 329-339.

${ }^{57}$ D. Di Tommaso and N.H. de Leeuw, J. Phys. Chem. B, 2008, 112, 6965-6975.

${ }^{58}$ S. Kerisit, A. Marmier and S.C. Parker, J. Phys. Chem. B, 2005, 109, 18211-18213.

${ }^{59}$ S.C. Parker, E.T. Kelsey, P.M. Oliver and J.O. Titiloye, Faraday Discuss., 1993, 95, $75-84$.

${ }^{60}$ J.J. Gilman, J. Appl. Phys., 1960, 31, 2208-2218.

${ }^{61}$ S.L.S. Stipp, Mol. Simul., 2002, 28, 497-516.

${ }^{62}$ J. Schutte, P. Rahe, L. Troger, S. Rode, R. Bechstein, M. Reichling and A. Kuhnue, Langmuir, 2010, 26, 8295-8300.

${ }^{63}$ J.S. Lardge, D.M. Duffy and M.J. Gillan, J. Phys. Chem. C, 2009, 113, 7207-7212. 
${ }^{64}$ K. Wright, R.T. Cygan and B. Slater, Phys. Chem. Chem. Phys. 2001, 3, 839-844.

${ }^{65}$ A. Rahaman, V.H. Grassian and C.J. Margulis, J. Phys. Chem. C, 2008, 112, $2109-$ 2115.

${ }^{66}$ N.H. de Leeuw and S.C. Parker, J. Phys. Chem. B, 1998, 102, 2914-2922.

${ }^{67}$ T.D. Perry IV, R.T. Cygan and R. Mitchell, Geochim. Cosmochim. Acta, 2007, 71, 5876-5887.

${ }^{68}$ S.L.S. Stipp, Geochim. Cosmochim. Acta, 1999, 63, 3121-3131.

${ }^{69}$ O.S. Pokrovsky, J.A. Mielczarski, O. Barres and J. Schott, Langmuir, 2000, 16, 2677-2688.

${ }^{70}$ M. Wolthers, L. Charlet and P. van Cappellen, Am. J. Sci., 2008, 308, 905-941.

${ }^{71}$ P. van Cappellen, L. Charlet, W. Stumm and P. Wersin, Geochim. Cosmochim. Acta, 1993, 57, 3505-3518.

${ }^{72}$ J.P.R. de Villiers, Am. Miner., 1971, 56, 758-767.

${ }^{73}$ S. Kerisit and S.C. Parker, J. Am. Chem. Soc., 2004, 126, 10152-10161. 